\title{
Stimuli-responsive polymers and their applications
}

Cite this: Polym. Chem., 2017, 8, 127 Menglian Wei, Yongfeng Gao, Xue Li and Michael J. Serpe*

Responsive polymer-based materials are capable of altering their chemical and/or physical properties upon exposure to external stimuli. These materials have been intensively studied over the years for a diverse range of applications, e.g., for on-demand drug delivery, tissue generation/repair, biosensing, smart coatings, and artificial muscles. Here, we review recent advances in the areas of sensing and biosensing, drug delivery, and actuators. Specific examples are given in each of these areas, and we highlight our group's work on poly( $\mathrm{N}$-isopropylacrylamide)-based microgels and assemblies.

\section{Introduction}

Polymers are ubiquitous in the things we use in everyday life, and are even responsible for life itself, e.g., due to the polymers of DNA and proteins. ${ }^{1}$ For ages, polymers have been used to improve the quality of life, although the true polymeric nature of the substances (e.g., natural rubber) was not known. ${ }^{2}$ Likely driven by the economic impetus of the rubber industry in the $19^{\text {th }}$ and $20^{\text {th }}$ centuries more effort was spent on investigating why the materials (i.e., polymers) behave the way they do. Hence, the intense debate about the nature and structure of polymers began, and eventually Staudinger's macromolecular theory was accepted, ${ }^{3,4}$ which described polymers as being

Department of Chemistry, University of Alberta, Edmonton, AB, T6G 2G2, Canada. E-mail:michael.serpe@ualberta.ca

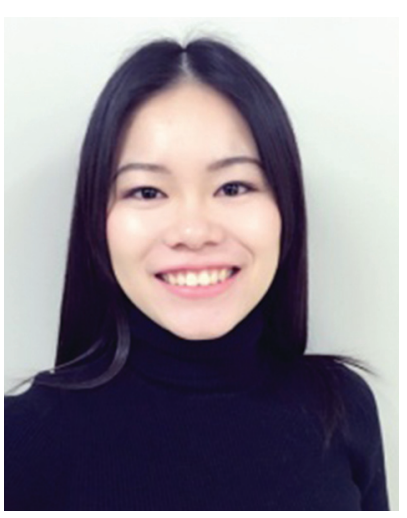

Menglian Wei
Menglian Wei received her B.Sc. degree from Wuhan University in 2011. She joined Prof. Michael J. Serpe's group as a Ph.D. student in 2012 and her research has focused on the development of a novel surface plasmon resonance spectrometer for studying. confined polymer brushes, and for improved sensing and biosensing. composed of many elementary units (monomers) covalently bound together. This theory was further supported by the experiments of Carothers, who first synthesized nylon., Following the work of these pioneers, a number of other researchers have made ground-breaking advances in the field, and the work has yielded many Nobel Prizes over the decades. This combined knowledge has allowed the development of polymers for nearly every application imaginable, and is single-handedly responsible for the high quality of life that many have become accustomed to.

This foundational research also led to the development of a new class of polymers, which respond to their environment by changing their physical and/or chemical properties. ${ }^{7-9}$ These polymers, referred to as stimuli-responsive polymers (or smart/ intelligent polymers), have been synthesized to be responsive to a variety of stimuli, e.g., $\mathrm{pH},{ }^{10}$ temperature,${ }^{11}$ mechanical force, ${ }^{12}$ the presence of various small molecules and

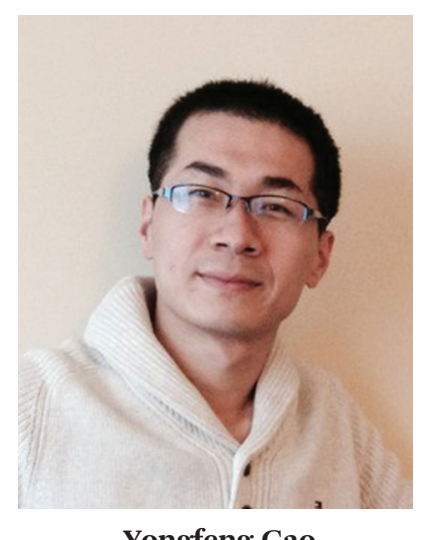

Yongfeng Gao
Yongfeng Gao received his B.Sc. degree in Chemistry from Tsinghua University, Beijing, China, in 2009 and obtained his M.Sc. degree in Chemical Engineering from Beijing University of Chemical Technology, Beijing, China, in 2012. He is presently a Ph.D. candidate in the Department of Chemistry at the University of Alberta, under the supervision of Prof. Michael J. Serpe. His research interests focus on stimuli-responsive microgels and microgel-based systems for controlled drug delivery. 
biomolecules, ${ }^{13}$ and electric/magnetic fields. ${ }^{14-16}$ Their development is oftentimes driven by the desire to mimic nature. ${ }^{17-19}$ Such intelligent polymers have found many applications in the fields of biology and medicine and can be used as sensors and biosensors, ${ }^{20}$ for controlled and triggered drug delivery, ${ }^{21}$ environmental remediation, ${ }^{22}$ chemo-mechanical actuators, ${ }^{23-25}$ and for many other applications. ${ }^{26-28}$

Of the multitude of responses to stimuli (some specifically mentioned above), by far the most well-studied and understood response is to temperature. For example, some polymers exhibit a lower critical solution temperature (LCST), ${ }^{29}$ which is the lowest temperature at which temperature induced demixing occurs. That is, below the LCST the polymer chains and solvent molecules are in one homogeneous mixed phase and above the LCST, phase separation occurs via an entropically driven process. Poly( $N$-isopropylacrylamide) (pNIPAm) $)^{11,30-32}$ is one of the most extensively studied temperature responsive polymers that exhibits a LCST at $\sim 32{ }^{\circ} \mathrm{C}$, which is close to the physiological temperature. As the solution temperature rises above the LCST, pNIPAm chains undergo a transition from an extended (solvated) random coil to a compact (desolvated) globular conformation. For individual polymer chains, the coil to globule transition can be thermodynamically controlled by adjusting the polymer composition, ${ }^{33}$ i.e., the LCST shifts to higher or lower temperature by copolymerization with a hydrophilic or hydrophobic monomer, respectively. ${ }^{34,35}$ There are a variety of polymers that exhibit LCSTs, such as poly[ $N-[2-$ (diethylamino)ethyl acrylamide]] (PDEAEAM), ${ }^{36} \operatorname{poly}(N, N$ dimethylaminoethyl methacrylate) (PDMAEMA), ${ }^{37-39} \operatorname{poly}(N, N$ diethylaminoethyl methacrylate) (PDEAEMA), ${ }^{40} \operatorname{poly}(2-(N-$ morpholine)ethyl methacrylate) (PMEMA), ${ }^{40}$ poly[oligo(ethylene glycol)methacrylate $]^{41,42}$ and $\operatorname{poly}(N, N$-diethylacrylamide $)$ (PDEAAM) ${ }^{43,44}$ In addition, multi-responsive polymers can be synthesized by incorporating other functional groups into the temperature responsive polymer. For example, $\mathrm{pH}$ responsive compounds that have ionizable functional groups capable of donating or accepting protons upon environmental $\mathrm{pH}$ changes could be used. Some common examples are, acrylic acid (AAc) ${ }^{10,45-47}$ and $N, N$-dimethylaminoethyl methacrylate (DMAEMA). ${ }^{48-50}$ Light responsive monomers can also be used to generate materials that exhibit both temperature and light responsivity; a common example is azobenzene. ${ }^{51-53}$ In most cases the response of these polymers is a result of light triggered isomerization of light sensitive molecules incorporated into the polymer, although other mechanisms are possible, including light triggered ionization. Finally, biologically responsive systems, e.g., enzyme responsive polymers, ${ }^{54-56}$ and glucose responsive polymers ${ }^{57,58}$ can also be generated, which have the ability to respond to stimuli that are inherently present in biological samples. ${ }^{59}$ Generally, the response is a result of capture biomolecules immobilized in the polymer interacting with the target, which results in network crosslinking and/or ionization.

In this review, we mainly discuss recent examples of the use of stimuli-responsive polymers for sensing and biosensing, controlled and triggered drug delivery, and artificial muscles. In these individual areas we will highlight recent work from our own group. We point out that this review will focus on applications, while many other recent reviews have focused on the synthesis and fundamental properties of stimuli responsive polymers, and those details will not be rehashed here. ${ }^{60-64}$

\section{Applications of stimuli-responsive polymers}

\subsection{Sensing and biosensing}

A sensor is a self-contained integrated device that is able to receive an input from its surroundings and convert it into an output signal that can be processed and converted to a readable result. ${ }^{65}$ Likewise, a biosensor is a device that is capable of detecting and quantifying biological species of interest.

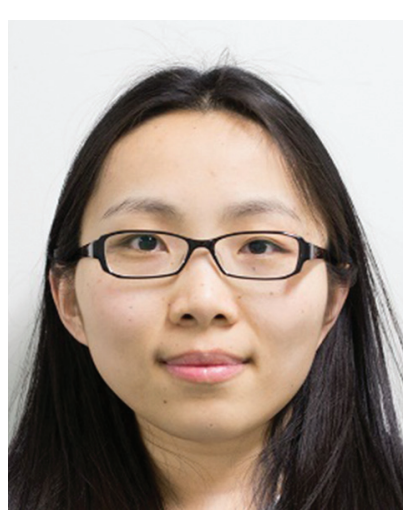

Xue Li
Xue Li received her B.S. degree from Qufu Normal University in 2009, and M.Sc. degree from Nanjing University in 2011 where she studied analytical chemistry in the group of Prof. $Y u$ Qin. In the same year she joined Prof. Michael J. Serpe's group as a Ph.D. student at the University of Alberta and received her Ph.D. in 2016. While in the Serpe group, she developed pNIPAm microgelbased etalons for biosensing applications and polymer-based materials for artificial muscles.

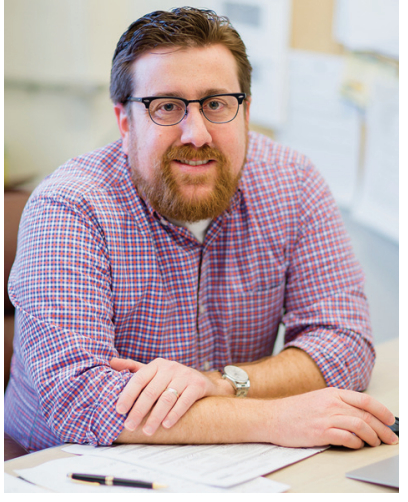

Michael J. Serpe

Michael J. Serpe received his B.S. degree from the University of Central Florida in 2000, his Ph.D. from the Georgia Institute of Technology in 2004 and completed his postdoctoral studies at Duke University in 2009. In the same year he joined the Department of Chemistry at the University of Alberta as an Assistant Professor and was promoted to Associate Professor in 2014. His group's research program is focused on developing new technologies to solve problems associated with health and the environment using fundamental and applied polymers, colloids (nano and microparticles), surface and materials chemistry. 
In general, a biosensor should be capable of detecting a species of interest (analyte) from a complex mixture containing a variety of interfering species, and provide accurate results in a short time. In some cases, biosensors should be able to detect biological analytes in resource-limited settings and at the point-of-care (POC) to increase efficiency of treating patients in developing parts of the world. Accomplishing this will have positive health outcomes for those people in developing parts of the world.

Responsive polymer-based sensors have attracted considerable attention due to their ability to convert the presence of analytes into a physical and/or chemical change that a user can relate to the status of a system. ${ }^{66}$ In one example, Dostalek and coworkers generated a poly( $\mathrm{N}$-isopropylacrylamide)-comethacrylic acid (pNIPAm-co-MAAc) hydrogel on a surface plasmon resonance (SPR) sensor surface with indium tin oxide microheaters embedded to allow for SPR signal tuning, as shown in Fig. 1. ${ }^{67}$ Using the microheaters, rapid thermal responses of the pNIPAm-based material between swollen and de-swollen could be triggered, yielding a thermo-optical coefficient of $\mathrm{d} n / \mathrm{d} T=2 \times 10^{-2}$ RIU K ${ }^{-1}$. Further, the hydrogel layer can serve as a 3D binding matrix for biosensor applications by engineering bio-recognition elements in the polymer network. In this example, the authors modified the hydrogel with mouse immunoglobulin G (mIgG) via 1-ethyl-3-(3-dimethylaminopropyl)carbodiimide (EDC) coupling, which was able to capture Alexa Fluor 647 dye-labeled goat antimouse IgG (a-mIgG). Enhanced fluorescence intensity was observed at the SPR resonance angle, which was attributed to the enhanced electric field by SPR. In addition, upon a temperature increase, the binding matrix collapsed and resulted in a resonance angle shift as well as diminished fluorescence intensity. Therefore, the fluorescence signal excited by SPR at the resonance angle can be virtually switched on and off by swelling and de-swelling of the hydrogel binding matrix.

Stimuli-responsive polymer-modified nanoparticles have attracted significant attention in recent years for sensing and biosensing. Due to quantum effects, nanoparticles exhibit

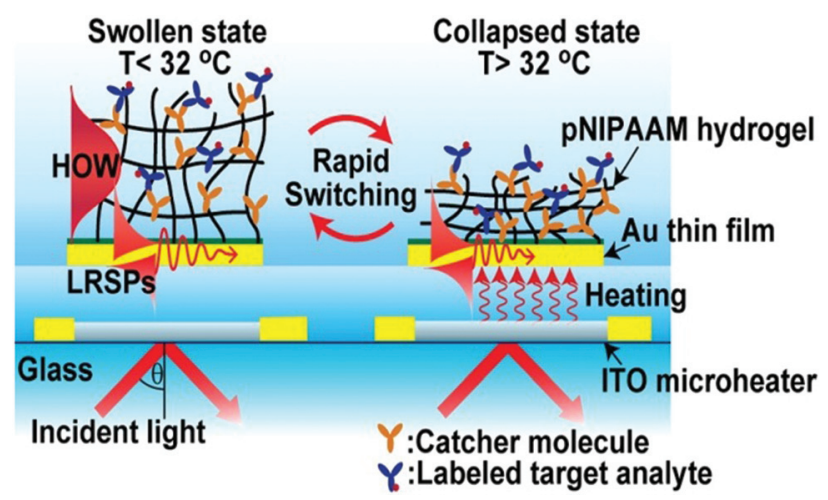

Fig. 1 Schematic diagram of a pNIPAm-co-MAAc hydrogel modified SPR sensor device with embedded ITO microheaters enabling rapid signal tuning. Reprinted with permission from ref. 67. Copyright 2013, American Chemical Society. unique optical properties (e.g., bright emission with no photobleaching), which make them ideal candidates for reporters in sensors. Furthermore, the conformational change of the surface bound stimuli responsive polymers can translate into observable optical property changes, which can be used for sensing and biosensing. In one example, Hoogenboom and coworkers developed a colorimetric temperature and salt sensor by coating Au nanoparticles (AuNPs) with a thermoresponsive pNIPAm shell. ${ }^{68}$ The pNIPAm polymer was synthesized by RAFT polymerization using methyl 2-(((butylthio)carbonothioyl)thio)propanoate (MBTTC) as a chain transfer agent (CTA), and further grafted to the AuNP surface by a ligand exchange process. When exposed to solutions of different salt concentrations, the pNIPAm modified AuNPs were able to change color from red to purple or blue at elevated temperatures, as shown schematically in Fig. 2. This is due to AuNP aggregation induced electronic coupling of the SPR leading to a visible color change. In this study, the authors also found that the range of temperature sensitivity increased in the presence of $\mathrm{NaSCN}$, compared to $\mathrm{NaCl}$, due to charge screening and Hofmeister effects.

A conceptually similar approach was used to generate $\mathrm{CO}_{2}$ sensors. $\mathrm{CO}_{2}$ has physiological significance, and abnormal concentrations have been associated with metabolism-related diseases. Ma et al. developed a $\mathrm{CO}_{2}$ sensor by coating AuNPs with the $\mathrm{CO}_{2}$ responsive polymer, poly( $N$-(3-amidino)-aniline) (PNAAN), as shown in Fig. 3c. ${ }^{69}$ The hybridized particles were synthesized by directly reducing $\mathrm{HAuCl}_{4}$ with the $\mathrm{CO}_{2}$ responsive monomer $\mathrm{N}$-(3-amidino)-aniline (NAAN). As $\mathrm{CO}_{2}$ dissolves in solution, the amidine group of PNAAN can be protonated into hydrophilic amidinium, which induced PNAAN to swell and detach from the AuNP surface, resulting in AuNP aggregation and a color change, as shown in Fig. $3 \mathrm{a}$ and b. The $\mathrm{CO}_{2}$ sensor exhibited a linear range of 0.0132 to $0.1584 \mathrm{hPa}$ and a limit of detection (LOD) of $0.0024 \mathrm{hPa}$ by monitoring the UV absorbance change of AuNPs.

In a very interesting application of nanotechnology combined with stimuli responsive polymers, Paek et al. generated a colorimetric $\mathrm{pH}$ sensor by anchoring "blue" and "orange" light emitting quantum dots (BQDs and OQDs) to a single graphene oxide (GO) sheet (MQD-GO) through different $\mathrm{pH}$ responsive linkers, as shown in Fig. $4 .^{70}$ The photoluminescence emissions of the BQDs and OQDs on MQD-GO can be controlled independently through the two types of pH-responsive linkers of poly(acrylic acid) (PAA) $\left(\mathrm{p} K_{\mathrm{a}}=4.5\right)$ and poly(2-vinylpyridine) $(\mathrm{P} 2 \mathrm{VP})\left(\mathrm{p} K_{\mathrm{a}}=3.0\right)$. The polymer linkers were able to change their conformation at distinct $\mathrm{pH}$ ranges, which ultimately tuned the Förster resonance energy transfer efficiencies from the BQDs to the GO and from the OQDs to the GO. As a result, the color of MQD-GO transitions from orange to near-white to blue over a wide range of $\mathrm{pH}$ values. Furthermore, the MQD-GO sensor showed excellent reversibility and high dispersion stability in pure water, all of which satisfy the critical requirements of a $\mathrm{pH}$ sensor.

Stimuli-responsive polymers can also be used as building blocks for generating photonic crystals (PCs), which exhibit 

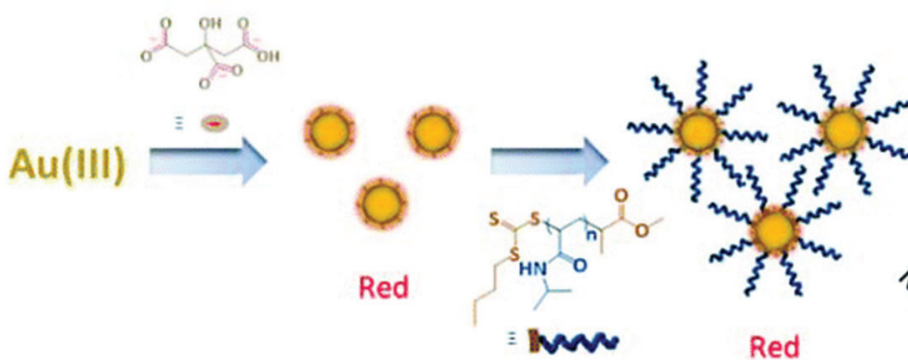

Red

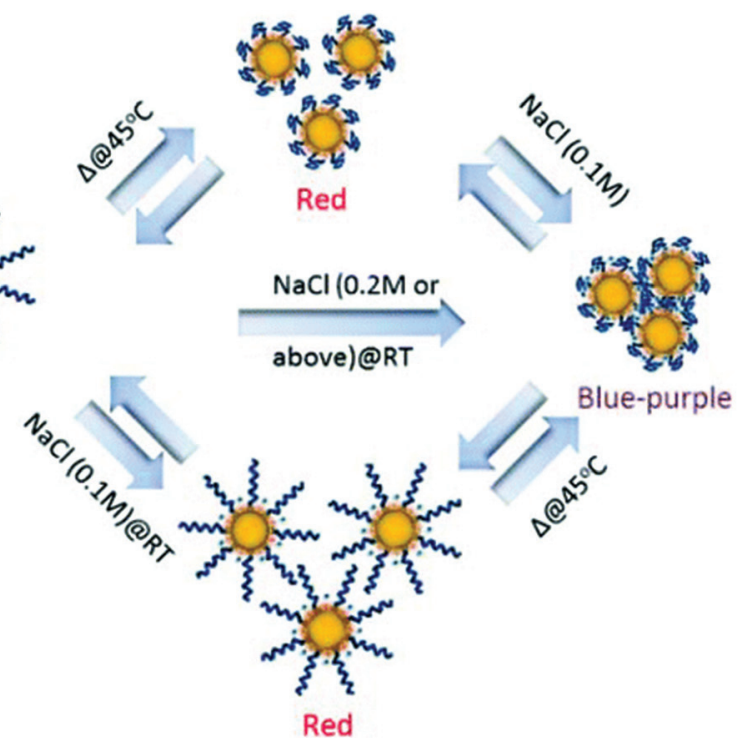

Fig. 2 Schematic illustration of the synthesis of pNIPAm brush modified AuNPs and their thermoresponsive behavior in aqueous solution and their aggregation in the presence of various concentrations of $\mathrm{NaCl}$. Reprinted with permission from ref. 68. Copyright 2016, The Royal Society of Chemistry.
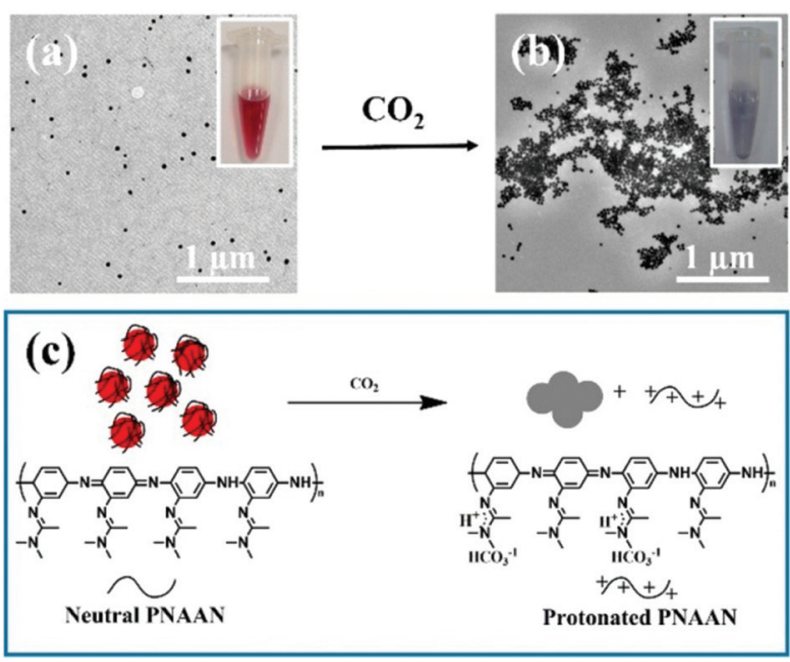

Fig. 3 TEM images of AuNPs (a) before and (b) after being purged with $\mathrm{CO}_{2}$ gas. (c) Schematic illustration of $\mathrm{CO}_{2}$ induced AuNP aggregation. Insets of (a) and (b) show photographs of the AuNP solutions (a) before and (b) after being purged with $\mathrm{CO}_{2}$ gas. Reprinted with permission from ref. 69. Copyright 2016, American Chemical Society.

structural color due to light interaction with the periodic structure of the material. These interactions ultimately lead to constructive/destructive interference of specific wavelengths of light, which yield color. Generally, PCs can be classified as one-dimensional (1-D), two-dimensional (2-D), and threedimensional (3-D) depending on the number of dimensions the order occurs, as shown in Fig. $5 .^{71}$ The wavelength of light "reflected" from the device is primarily determined by the material's lattice spacing, which also dictates the visual color of the material. If the photonic crystal is composed of respon- a)

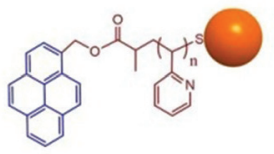

b)

c)
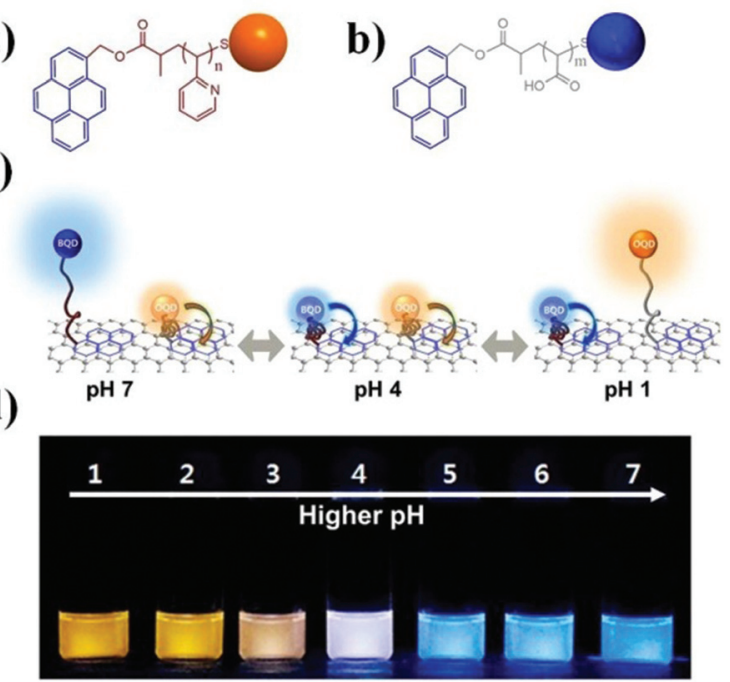

Fig. 4 Structures of (a) P2VP-OQD and (b) PAA-BQD. (c) Schematic illustration of the conformation and behavior of MQD-GO at the indicated solution $\mathrm{pH}$. (d) Photographs of MQD-GO in buffer solutions at the indicated solution $\mathrm{pH}$ under irradiation with $365 \mathrm{~nm}$ light. Reprinted with permission from ref. 70. Copyright 2014, American Chemical Society.

sive polymer building blocks, then the lattice spacing (and visual color) can also be tuned by applying external stimuli. Perhaps the most well-known early examples of responsive PCs come from the Asher group who pioneered the development of 3-D photonic crystal-based sensors. This was accomplished by embedding ordered crystalline colloidal arrays (CCA) in analyte-responsive hydrogel matrices. These materials were used to detect solution $\mathrm{pH}$, ionic strength, temperature, and 


\section{Photonic Crystals}

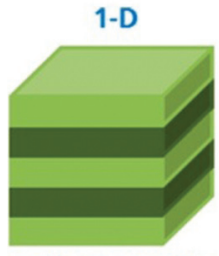

Periodic structure

in one direction

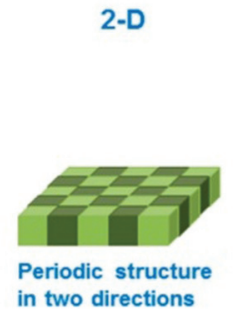

Fig. 5 Schematic illustrating the different dimensions of order in photonic materials. Reprinted with permission from ref. 71. Copyright 2014, American Chemical Society.

the presence of biological or chemical targets. ${ }^{72-78}$ In one example, a 3-D PC sensor for metal ions was developed by copolymerizing 4-acryloylaminobenzo-18-crown-6 (AAB18C6) into a hydrogel composed of an ordered array of polystyrene spheres. $^{72}$ As metal ions bound to the crown ether of AAB18C6, the hydrogel swelled due to the charge density increase in the polymer network yielding an increase in osmotic pressure and network swelling. As a result of the swelling, the polystyrene spheres in the hydrogel network separated from one another resulting in a red shift of the "reflected" light.

In another example, Hayward and coworkers developed a colorimetric temperature sensor by depositing alternating layers of high and low refractive index polymers on a surface to generate a one dimensional Bragg mirror as shown in Fig. $6 \mathrm{~b} .^{79}$ The high refractive index polymer was non-responsive poly(para-methyl styrene) (PpMS), while the low refractive index polymer was temperature responsive poly( $N$-isopropylacrylamide)-co-acrylic acid. After immersion in water at room

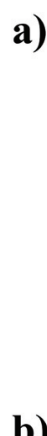

b)
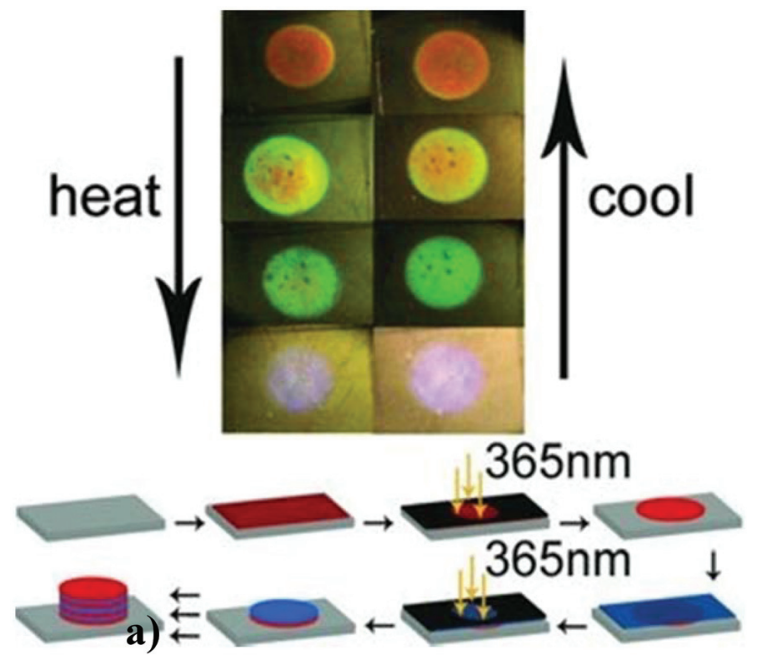

Fig. 6 (a) Photographs of a sensor changing color as a function of temperature-heating induces deswelling, while cooling induces swelling. (b) Schematic representation of sensor fabrication (red layers, PpMS; blue layers, pNIPAm-co-AAc). Reprinted with permission from ref. 79. Copyright 2012, Wiley-VCH Verlag GmbH \& Co. KGaA. temperature, the polymer stack exhibited a Bragg peak at $710 \mathrm{~nm}$ and an orange color. Upon heating, the pNIPAm-based polymer layer shrunk, resulting in the approach of the PpMS layers and a concomitant shift in the reflected light to lower wavelengths. This also yielded a color change, as can be seen in Fig. 6a. Furthermore, the color tunability was fully reversible with temperature.

The Serpe group has fabricated etalons (a basic 1D photonic material) that respond to a variety of stimuli, such as $\mathrm{pH},{ }^{80}$ light, ${ }^{81}$ electric field, ${ }^{82}$ temperature, ${ }^{46}$ nerve agents, ${ }^{83}$ and various biomolecules. ${ }^{84}$ As detailed in these publications, etalons were constructed by depositing a thin layer of Au (typically $15 \mathrm{~nm}$ ) on top of a glass substrate by thermal evaporation followed by "painting" a layer of microgels onto the $\mathrm{Au}$, and subsequent deposition of another Au layer (typically $15 \mathrm{~nm}$ ) on top of the microgel layer. This structure allows light to enter the dielectric cavity and resonate between the two reflective Au layers. This resonating light yields constructive and destructive interference, allowing certain wavelengths of light to be reflected. The wavelength of the reflected light can be predicted using eqn (1):

$$
\lambda m=2 n d \cos \theta
$$

where the specific wavelength maximum of the reflected peak $(\lambda)$ depends on the peak order $(m)$, refractive index of the dielectric $(n)$ and the spacing between the mirrors $(d)$, as well as the angle of incidence $(\theta)$. For etalons, the Au and pNIPAmbased microgel layers serve as the mirrors and the dielectric layer, respectively. These devices have been shown to be able to detect glucose, ${ }^{85} \mathrm{CO}_{2},{ }^{86}$ triacylglycerols (TAGs), ${ }^{87}$ proteins, ${ }^{88}$ and DNA. ${ }^{89}$ For example, Zhang et al. ${ }^{87}$ synthesized TAGresponsive microgels by conjugating lipase to $\operatorname{poly}(N$-isopropylacrylamide- $\mathrm{Co}$-4-vinylpyridine- $\mathrm{Co}$ - $\mathrm{N}$-acryloxysuccinimide) (pNIPAm-4VP-NAS) microgels, as shown in Fig. 7. When triolein penetrated into the microgel layer, it was hydrolyzed into a long chain fatty acid $\left(\mathrm{C}_{18} \mathrm{H}_{37} \mathrm{COOH}\right)$ by the lipase inside the microgels. The long chain fatty acid could subsequently attach to the microgels via acid-base reactions between fatty acid and the microgel's pyridine groups. The lipophilicity of long chain fatty acid increases the hydrophobicity of the microgels, hence water was expelled from the microgels, and they collapsed. This resulted in a blue shift of the etalon's reflectance peaks, which corresponded to the concentration of triolein (Fig. 7). Such devices were able to detect the physiologically relevant range of triolein.

Multi-responsive microgels and etalons could also be generated by incorporating a multi-responsive moiety into the pNIPAm-based microgel at the time of polymerization. ${ }^{83,90}$ Serpe and coworkers synthesized spiropyran (SP)-modified pNIPAm-based microgels and investigated their responsivity to multiple stimuli including temperature, $\mathrm{pH}, \mathrm{Cu}^{2+}$, UV, visible, and near-infrared radiation. ${ }^{90}$ The responses were a result of the SP groups undergoing a reversible isomerization/reaction from a neutral to a charged form, as shown in Fig. 8. SP's $\mathrm{C}($ spiro $)-\mathrm{O}$ bond can be cleaved by UV light exposure or at low 
a)

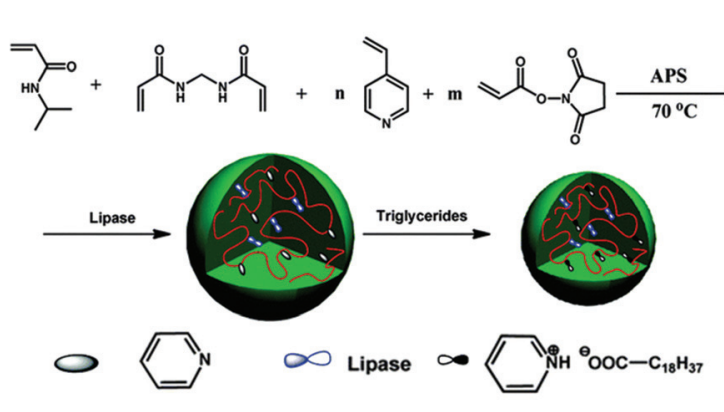

b)

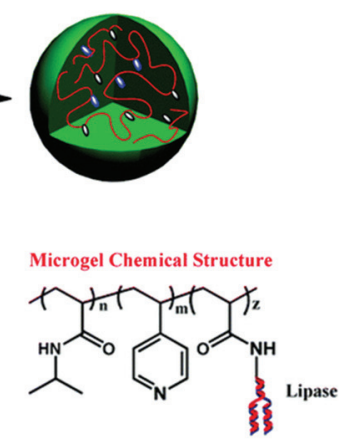

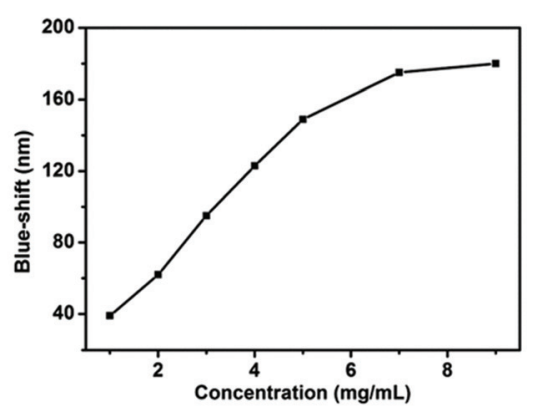

Fig. 7 (a) Triacylglyceride responsive microgel synthesis scheme, and the resulting microgel chemical composition. (b) The magnitude of the reflectance peak blue-shift as a function of triglyceride concentration at $30{ }^{\circ} \mathrm{C}$. Reprinted with permission from ref. 87 . Copyright 2015 , The Royal Society of Chemistry.

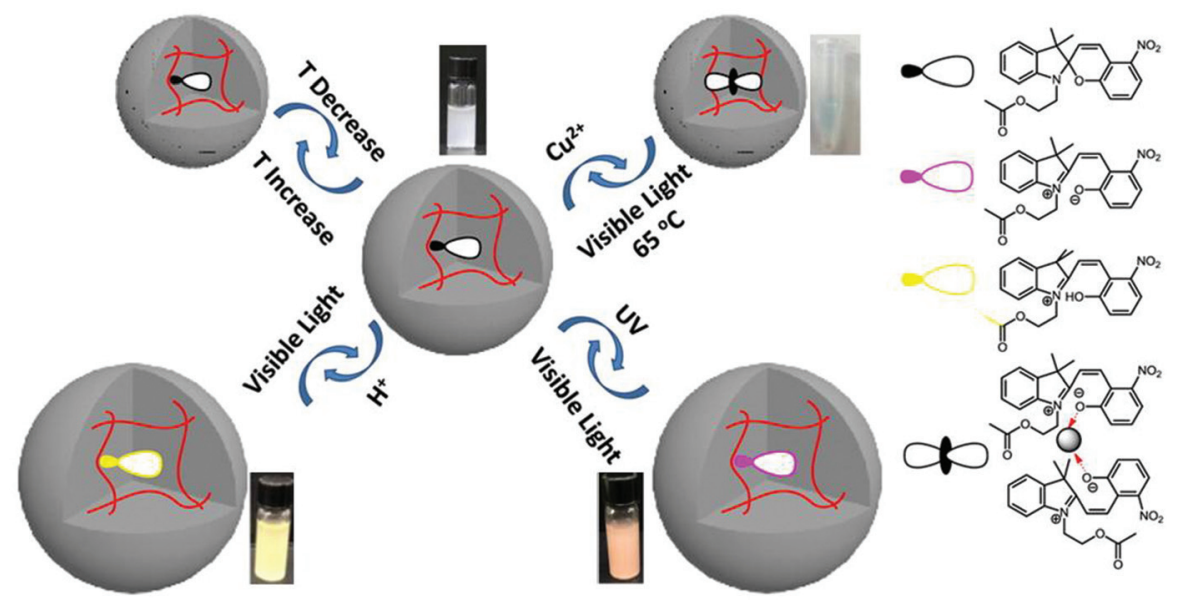

Fig. 8 Spiropyran modified pNIPAm-based microgel response to multiple stimuli. Reprinted with permission from ref. 90. Copyright 2015, American Chemical Society.

$\mathrm{pH}(<4)$. During the process, SP isomerizes from a colorless, nonplanar, closed and neutral form to the colored, planar, open and charged merocyanine form (MC), which increases the microgel's inner osmotic pressure and results in microgel swelling. MC can also be triggered to reform SP by exposure to visible light. The $\mathrm{C}\left(\right.$ spiro)-O bond can be cleaved by $\mathrm{Cu}^{2+}$ and subsequently bind to $\mathrm{Cu}^{2+}$ through phenoxide anions in the microgel, which result in microgel collapse. The spiropyranmodified microgels retained their responsivity after incorporation into etalons, which allows their optical properties to be tuned upon exposure to these various stimuli.

While stimuli-responsive polymers have a great potential to be applied for sensing and biosensing, there are still many challenges that lie ahead. For example, there is always room to improve sensor sensitivity, selectivity, stability, sampling, and signal readout mechanisms. However, we believe that the benefits of stimuli-responsive-based sensors (i.e., their ease of fabrication, and versatility) far outweigh the negatives, and one will find these sensors being employed in evermore diverse environments.

\subsection{Controlled drug delivery}

Living systems react to external stimuli by responding and adapting to changing external conditions. Polymer scientists have been trying to mimic this behavior by creating so called smart polymers. Smart polymers have shown promise for biomedical applications, and have found use as controlled/ triggered/targeted drug delivery vehicles, tissue engineering scaffolds, cell culture supports, bioseparation apparatus, sensors, and actuators/artificial muscles. The concept of stimuli-responsive polymer-based drug delivery systems was first reported in the late 1970s with the use of thermosensitive liposomes for the local release of drugs via hyperthermia. ${ }^{91}$ Subsequently, a great deal of research has been carried out on stimuli-responsive materials for drug delivery, especially on the design and application of responsive polymers. ${ }^{13,66,92-94}$ The design of new systems and approaches must meet the challenges associated with administration in the body. The systems must be: (i) simply administered; (ii) capable of delivery to the desired locations in response to a stimulus; and 
(iii) composed of non-toxic, biocompatible and biodegradable components. A variety of stimuli-responsive polymer-based materials have been used for this application, including crosslinked gel networks, and non-crosslinked block copolymer assemblies.

One of the most important classes of stimuli-responsive polymers used for controlled drug delivery is crosslinked polymer networks, e.g., hydrogels and microgels. Hydrogels have been shown to be useful for a wide range of biomedical applications because of their porous structure and water swellability. Their porosity permits loading of drugs into the gel matrix and subsequent drug release at a rate dependent on the diffusion coefficient of the small molecule or macromolecule in the gel network. In addition, many hydrogels can change their degree of swellability in response to changes in their environment as mentioned earlier. Based on the structures and properties of hydrogels, most of them have been used for transdermal drug delivery or injectable drug delivery systems. For transdermal drug delivery, microneedles improve drug permeability into skin by providing a clear entry pathway; this improves the delivery efficiency of vaccines and pharmaceutical agents (such as small molecules, protein, DNA). ${ }^{95-97}$ Compared to the solid microneedles, ${ }^{98}$ hydrogel-forming microneedle arrays can rapidly imbibe skin interstitial fluid to form discrete in situ hydrogel bulbs to control drug administration at a rate faster than traditional patch systems used for controlled drug release. The most important feature for hydrogel-forming microneedle arrays is the swelling induced release mechanism. ${ }^{99}$ By incorporating stimuli-responsive units into microneedles, one can trigger on-demand drug release by manipulating external stimuli. ${ }^{100,101}$ In one example, the McCoy group combined hydrogel-forming microneedles and light responsive drug conjugates to generate novel devices for on-demand transdermal drug delivery. ${ }^{102}$ For this device, poly(2-hydroxyethyl methacrylate) (HEMA) was used and crosslinked by ethylene glycol dimethacrylate (EGDMA). Microneedles were generated utilizing micromolding, and exhibited desirable mechanical properties. The devices were shown to release ibuprofen over a prolonged period of time (up to $160 \mathrm{~h}$ ) in response to light. The release could also be turned on and off by turning the light on and off, respectively. By mimicking the endoparasite Pomphorhynchus laevis that attaches to its host's intestinal wall, the researchers developed a biphase microneedle array that mechanically interlocks with tissue through swellable microneedle tips to increase adhesion strength. ${ }^{103}$ The light responsive units incorporated into the microneedles yielded patches with light triggered release properties to provide photothermal therapy and chemotherapy. ${ }^{104,105}$ The light-activated heating and releasing behavior can be precisely controlled and switched on and off on demand for several cycles.

Recently, the $\mathrm{Gu}$ group also developed microneedle arraybased patches with responsive properties for smart insulin ${ }^{106,107}$ and controlled antibody ${ }^{108}$ delivery. A novel glucose-responsive insulin delivery device was reported based on microneedle array patches integrated with hypoxia-sensitive hyaluronic acid (HS-HA) vesicles containing insulin and glucose oxidase (GOx). By taking advantage of local generation of hypoxia, they trigger the insulin release in response to hyperglycemia. This hypoxia responsive transduction was achieved by converting hydrophobic 2-nitroimidazole to hydrophilic 2-aminoimidazoles in a hypoxic environment. The design of the microneedle array patches are shown in Fig. 9. The amphiphilic hyaluronic acid was synthesized and used to form self-assembled vesicles, which encapsulate both human insulin and GOx. When the blood glucose level is high, the dissolved oxygen is rapidly consumed due to the glucose oxidation reaction. This produces a local hypoxic environment, which reduces 2-nitroimidazole, resulting in the dissociation of vesicles and subsequent release of insulin.

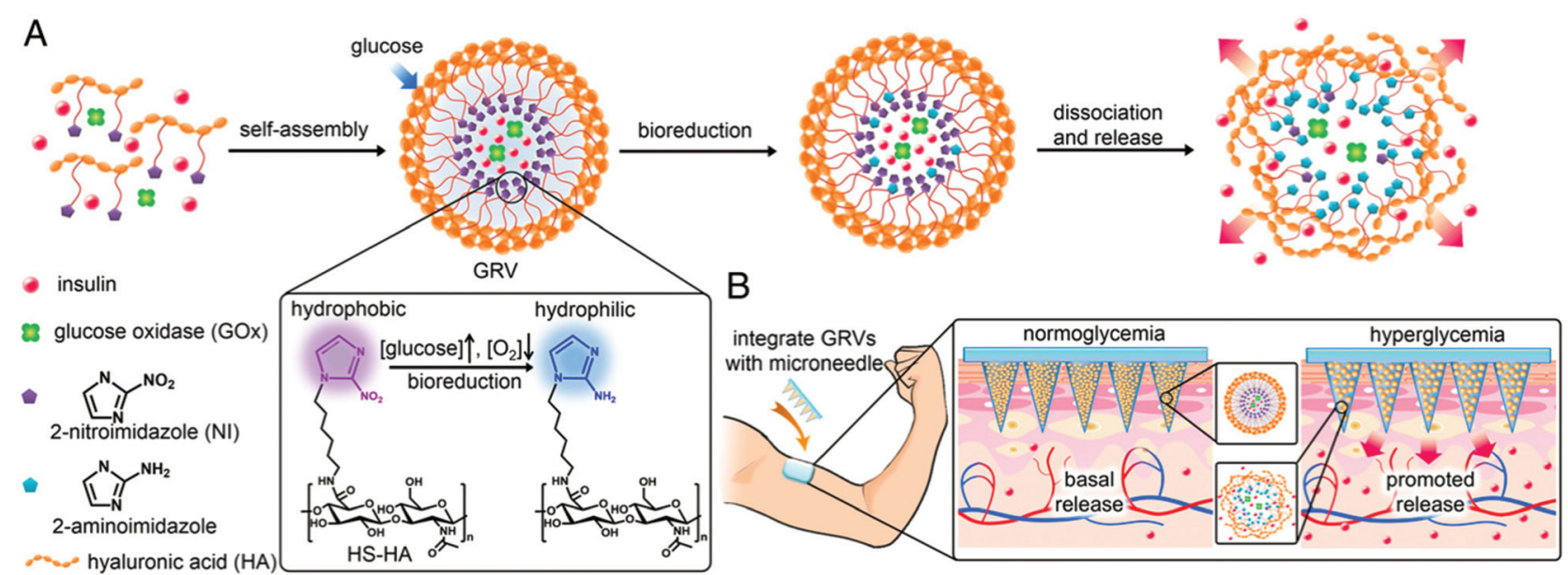

Fig. 9 Schematic depiction of the glucose-responsive insulin delivery system using hypoxia-sensitive vesicle-loading $\mathrm{MN}$-array patches. (A) Formation and mechanism of glucose responsive vesicles composed of HS-HA. (B) Schematic of the GRV-containing MN-array patch (smart insulin patch) for in vivo insulin delivery triggered by a hyperglycaemic state to release more insulin. Reprinted with permission from ref. 107. Copyright 2015, National Academy of Sciences. 
Furthermore, the vesicle was integrated into the microneedle array and can be used for the painless and continuous administration of insulin. This smart insulin patch with its novel triggering mechanism may offer the possibility of closed-loop delivery of insulin. It will also guide the development of a useful drug delivery platform for treating other diseases using artificial vesicles. In further studies, they also investigated the integration of microneedles with pancreatic cells and synthetic glucose-signal amplifiers for smart insulin delivery. Importantly, for the first time, a synthetic amplifier was incorporated to quickly amplify the physiological signal, in this case "glucose level," for effective transport of the signal and sufficient stimulation of insulin secretion from the $\beta$-cells.

Similar to bulk gels, microgels can also be made biocompatible, however, due to their small size, they exhibit many advantages over bulk gels when used as biomaterials. One major advantage is that the rate of microgel response to external stimuli is much faster than bulk gels. ${ }^{93,109}$ They can also be modified chemically such that they circulate in the blood stream for long periods of time, and are biodegradable for sustained release. The degradability also allows the microgels to be quickly cleared from the body. In addition, microgels can be used as building blocks for the fabrication of biomedical devices with improved and/or new function. ${ }^{110}$ These many advantages make microgels ideal candidates for building controlled drug delivery systems. ${ }^{62,93,94,109-111}$ As a drug carrier, pNIPAm-based microgels combine the advantages of both hydrogels and nanoparticles. PNIPAm microgel particles have a sponge-like structure with interstitial spaces filled with water. Drug molecules can be loaded by equilibrium partitioning between the solution and microgel phases. Electrostatic interaction, hydrophobic interaction, and/or hydrogenbonding may play an important role for drug loading. The Serpe group developed a novel microgel-based assembly (reservoir device) as a new platform for drug delivery. ${ }^{112-116}$ The structure of this device and the release mechanism is shown in Fig. 10. The device is usually composed of a pNIPAm-co-AAc microgel layer sandwiched between two thin Au layers (all on a glass support) and was used as a novel platform for controlled and triggered small molecule delivery. Tris(4-(dimethylamino) phenyl)methylium chloride (crystal violet, CV), which is positively charged, was loaded into the microgel layer of the device and released in a $\mathrm{pH}$ dependent fashion, at a rate that could be controlled by the thickness of the $\mathrm{Au}$ layer coating the microgel. ${ }^{112}$ The model drug could be released in an "on-off" fashion, by systematically varying the solution $\mathrm{pH}$. Furthermore, by modifying the top layer Au surface, we can control the drug release with a lower thickness of Au. ${ }^{113}$

In another example, the release of small molecules can be controlled by the aggregation state of microgels. For example, by combining two oppositely charged microgels, we can control the microgels' aggregation behavior to control drug release. In this example, microgels copolymerized with acrylic acid exhibit a negative charge above $\mathrm{pH} 4.25$, while the microgels copolymerized with $N$-[3-(dimethylamino)propyl]methacrylamide exhibit a positive charge below pH 8.4; these microgels are neutral outside of these $\mathrm{pH}$ ranges. We show that aggregates form when the two independent sets of microgels were exposed to one another in a solution that renders them both charged. In solutions of $\mathrm{pH}$ outside of this range, the microgels disaggregate because one of the microgels becomes neutralized. This behavior was exploited to load (aggregation) and release (disaggregation) a small-molecule model drug. ${ }^{115}$ This aggregate based system provides evidence of how the charged pNIPAm-based microgels are applied in controlled/ triggered drug release.

Recently, we showed that a single microgel-based reservoir device could be used for sequential and controlled release of more than one small molecule to a system. ${ }^{114}$ By incorporating microgels with different chemistries that allow them to be ionized at different solution $\mathrm{pH}$ into a single reservoir device, the release of small molecules and their dosages can be controlled and tuned. This is shown in Fig. 11. Specifically,
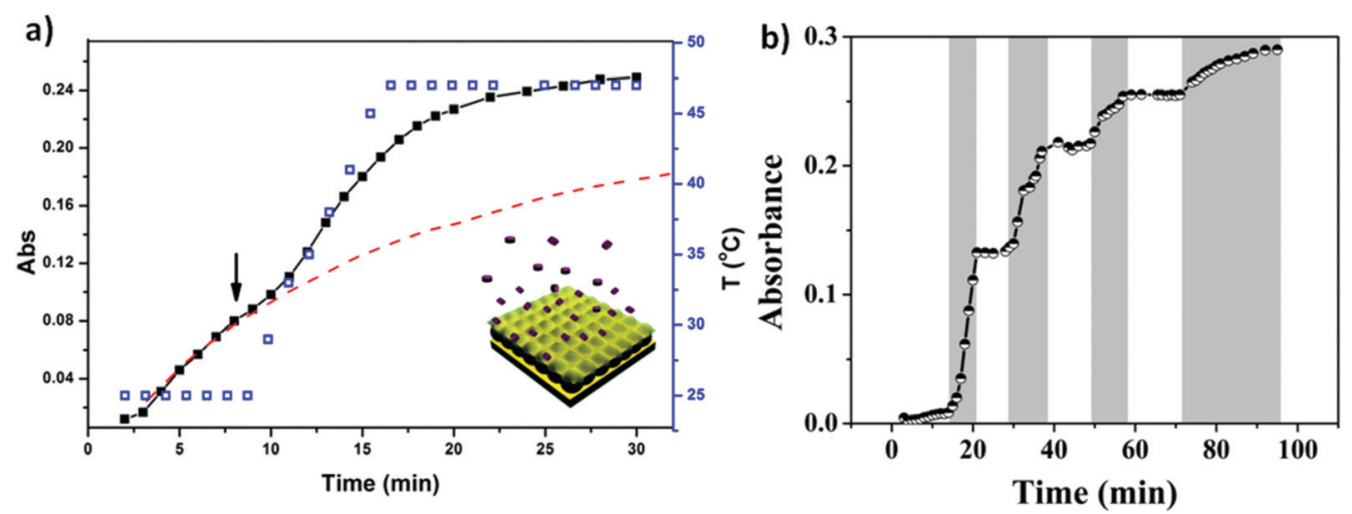

Fig. 10 The structure of microgel-based assemblies and their (a) temperature and (b) pH triggered release profile with a $500 \mathrm{~nm}$ Au overlayer. For (a), the absorbance was monitored at $590 \mathrm{~nm}$, (ロ) corresponds to the release profile (left $y$-axis), ( $\square$ ) corresponds to the temperature profile (right $y$-axis), while the dashed line shows the predicted release profile if the temperature wasn't increased. The shaded regions in (b) indicate where the solution $\mathrm{pH}$ was 3.0, while the white regions indicate where the solution $\mathrm{pH}$ was 6.5 . The solution $\mathrm{pH}$ was varied by adding either $\mathrm{HCl}$ or $\mathrm{NaOH}$. Reprinted with permission from ref. 112. Copyright 2013, American Chemical Society. 
(a)

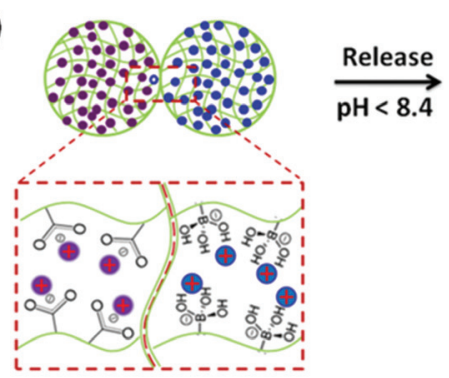

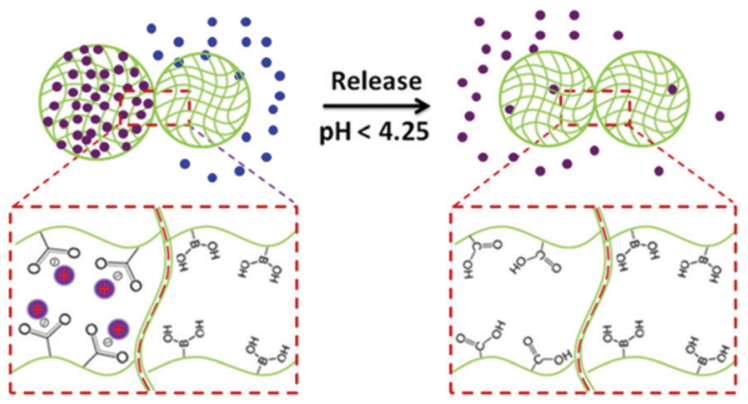

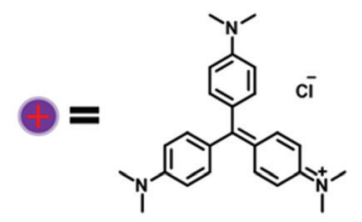

(b)
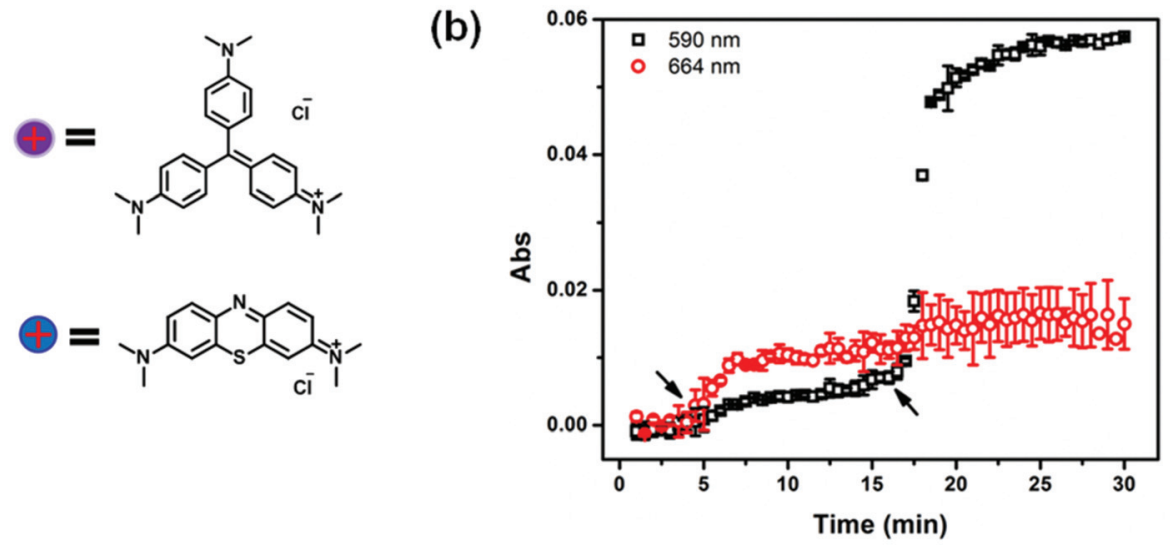

Fig. 11 (a) Schematic illustration of $\mathrm{pH}$ triggered MB and CV release from APBA-MG and AAC-MG, respectively. As each microgel is neutralized, the electrostatic interactions between the microgel and the charged molecules are diminished, and the MB and CV are released from the microgel sequentially. (b) The release profile for a device made of APBA-MG and AAC-MG loaded with MB and CV, respectively. The arrows indicate the times that the solution $\mathrm{pH}$ was changed to 7 (at $\sim 5 \mathrm{~min}$ ) and 3 (at $\sim 17 \mathrm{~min}$ ). Two different wavelengths were monitored (590 nm and $664 \mathrm{~nm}$, for $\mathrm{CV}$ and MB, respectively) that correspond to the absorbance of the two different model drugs. Reprinted with permission from ref. 114. Copyright 2016, The Royal Society of Chemistry.

pNIPAm-co-AAc microgels (AAc-MG) and poly( $N$-isopropylacrylamide-3-(acrylamido)phenylboronic acid) (pNIPAm- $\mathrm{co}$ APBA) microgels (APBA-MG) were used to construct reservoir devices and were loaded with the model drugs CV and methylene blue (MB), respectively. These two model drugs were chosen because of their strong visible light absorption and their positive charge, which allows them to interact strongly with the negatively charged microgels. At $\mathrm{pH} 10.0, \mathrm{CV}$ and $\mathrm{MB}$ (both positively charged) exhibited strong electrostatic interactions with both the negatively charged AAc and APBA-modified microgels, respectively. At pH 7.0, the APBA groups were neutralized, allowing $\mathrm{MB}$ to be released from the APBA-MG only. When the solution $\mathrm{pH}$ was again lowered to 3.0, the AAc groups are neutralized allowing $\mathrm{CV}$ to be released from the AAc-MG. These systems represent a versatile approach to sequentially delivering small molecules to a system, in a triggered fashion, with tunable release kinetics. Importantly, their release behavior can be easily tuned by simply changing the microgel chemistry, e.g., by generating reservoir devices from microgels that ionize at different solution $\mathrm{pH}$. This would allow one to deliver various small molecules to a system triggered by a variety of solution $\mathrm{pH}$. This, combined with the tunable release kinetics and the ability to array these devices on a single substrate, make this delivery platform extremely versatile, powerful, and unique.
Another important stimuli-responsive polymer architecture used in controlled drug delivery systems are block copolymer self-assembled structures, such as liposomes, micelles, and vesicles. Temperature responsive polymeric micelles have attracted considerable attention due to their sharp change of properties in response to a small change in environmental temperature. When using a temperature responsive polymer as the hydrophobic core-forming segment, a core-shell micelle structure forms above its LCST due to the hydrophobic interaction among the dehydrated polymer chains. ${ }^{117-120}$ For example, Discher and Yang ${ }^{121}$ used poly (ethylene oxide)-block-poly( $N$-isopropylacrylamide) (PEO- $b$ pNIPAm) block copolymers to generate micelles capable of releasing small molecules as a function of temperature. The polymers become amphiphilic in water above body temperature $\left(37^{\circ} \mathrm{C}\right)$ and self-assemble into micelles that can encapsulate both hydrophilic and hydrophobic molecules. With a decrease in temperature, the micelles disassemble and release their contents.

Photochromic molecules have also been widely utilized in responsive materials to build light responsive polymer systems. The Liu group reported the fabrication of photochromic polymersomes exhibiting photoswitchable and reversible bilayer permeability from newly designed poly(ethylene oxide)$b$-PSPA (PEO- $b$-PSPA) diblock copolymers, where SPA is a 
spiropyran (SP)-based monomer containing a unique carbamate linkage. ${ }^{122}$ Upon self-assembly into polymersomes, SP moieties within vesicle bilayers undergo reversible photo-triggered isomerization between the hydrophobic spiropyran (SP, $\lambda_{2}>450 \mathrm{~nm}$ irradiation) and zwitterionic merocyanine (MC, $\lambda_{1}<420 \mathrm{~nm}$ irradiation) states. The microstructures of both SP and MC polymersomes are synergistically stabilized due to cooperative noncovalent interactions from hydrophobic, hydrogen bonding, $\pi-\pi$ stacking, and paired electrostatic (zwitterionic) interactions, with the latter two types being exclusive for MC polymersomes. Moreover, reversible phototriggered SP/MC polymersome transition is accompanied by membrane permeability switching from being impermeable to selectively permeable towards non-charged, charged, and zwitterionic small molecule species below critical molar masses, as shown in Fig. 12. They further demonstrate photoswitchable spatiotemporal release of 4',6-diamidino-2-phenylindole (DAPI, cell nuclei staining dye) within living HeLa cells. UV-actuated MC polymersomes possess two types of release modules: (1) sustained release upon short UV irradiation duration by taking advantage of the unexpectedly slow spontaneous MC-to-SP transition kinetics $\left(t_{1 / 2}>20 \mathrm{~h}\right)$ in the dark; and (2) on-demand and switchable release under alternated UV-vis light irradiation. In another example, the Huang group incorporated azobenzene derivatives into supramolecular structures to create a supra-amphiphilic polypseudorotaxane, which can self-assemble to form vesicles in water. ${ }^{123}$ Due to the dualresponsivity of the molecular recognition motif, the reversible transformations between solid nanospheres based on the selfassembly of the polymer backbone and vesicles based on the self-assembly of the supra-amphiphilic polypseudorotaxane was achieved by adjusting the solution temperature or UV- visible light exposure. These dual-responsive aggregation behaviors were used to study the controlled release of water-soluble dye calcein molecules.

Disulfide bonds are well known to exhibit a high propensity to be reduced into thiols, and have been proposed as a candidate to develop smart responsive systems for controlled drug delivery. Zhu and coworkers synthesized multiblock copolymers with multiple enes and disulfides in hydrophobic blocks and then encapsulated modified doxorubicin (Dox) via conjugation and core-crosslinking reactions. ${ }^{124}$ The obtained nanoprodrug micelles presented stable nano-scaled spherical particles under physiological conditions, while quickly dissociating in response to $10 \mathrm{mM}$ D,L-dithiothreitol (DTT). The Oh group also recently reported a novel rosin-based block copolymer designed to self-assemble toward micellar nanocarriers with positioned disulfides at interfaces of hydrophobic rosin cores and hydrophilic poly(ethylene glycol) (PEG) coronas. ${ }^{125}$ This block copolymer-based self-assembled micellar nanocarrier exhibited glutathione (GSH)-responsivity that enhanced release of encapsulated drugs. The resulting micelles with PEG coronas had excellent colloidal stability in the presence of proteins, suggesting prolonged blood circulation in vivo. In response to $10 \mathrm{mM} \mathrm{GSH}$ as a biological reducing agent, the disulfides at the core/corona interfaces were effectively cleaved, causing the destabilization of micelles, thus leading to the enhanced release of encapsulated Dox. Due to the hydrophobicity of rosin species in micellar cores, the Dox release appeared to be slowed. These studies suggest that the designing of responsive block copolymers and their assembled nanostructures with reduction-responsive properties offers great versatility as intracellular drug-delivery nanocarriers for cancer therapy.

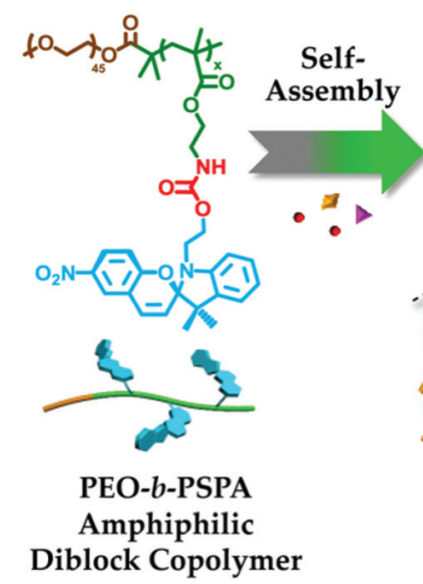

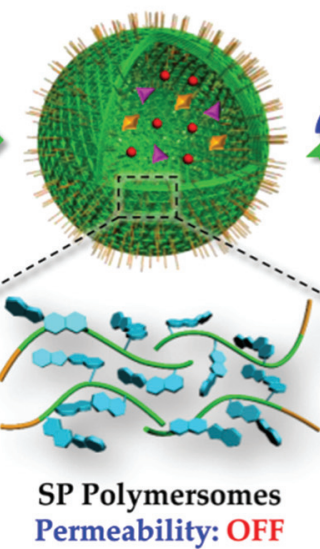

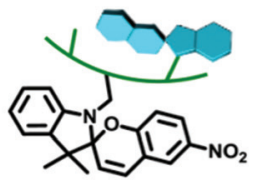

Hydrophobic SP Moieties

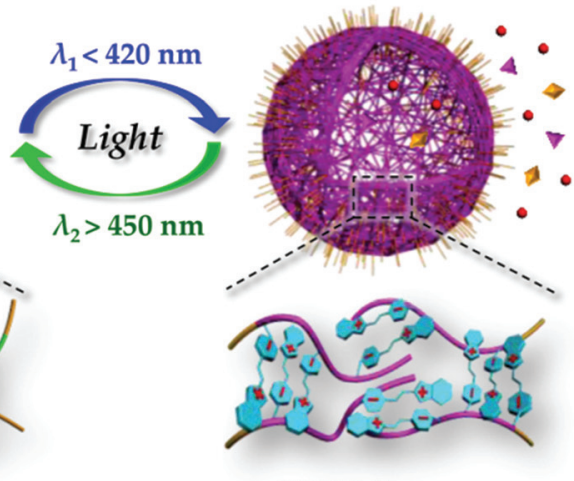

MC Polymersomes Selective Permeability: ON
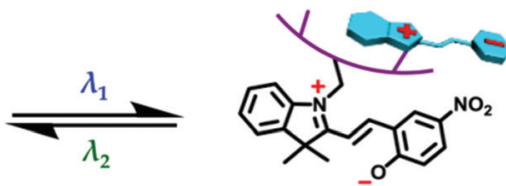

Zwitterionic MC Moieties

Fig. 12 Photochromic polymersomes exhibiting photoswitchable and reversible bilayer permeability. Reprinted with permission from ref. 122. Copyright 2015, American Chemical Society. 
The wide range of stimuli that can be exploited to trigger drug release at the right place and time, and the diversity of responsive materials and other functional materials that can be assembled in different architectures, allow great flexibility in the design of stimuli-responsive drug delivery systems. As we have shown in this section, building up novel structures from stimuli-responsive polymers can yield materials with important applications to solve problems related to human health. The next section will describe their utility as artificial muscles and actuators.

\subsection{Artificial muscles and actuators}

Natural muscles are biological organs that transform chemical energy into mechanical energy. The process is complex, and involves an electrical pulse from the brain that triggers the liberation of ions inside the sarcomere, chemical reactions (ATP hydrolysis), and eventual conformational changes along the natural muscle fibers. For years, groups have been attempting to mimic this behavior, and Otero, ${ }^{126,127}$ Inganäs, ${ }^{128,129}$ and MacDiarmid $^{130,131}$ have conducted numerous investigations on bilayers (the most common polymer-based actuator format) composed of a single actuating conducting polymer film deposited on an electromechanically inert layer. In a few examples, artificial muscles with tactile sensitivities were constructed from electrochemo-mechanical and macroscopic devices using films of PPy electrogenerated on double-sided tape to generate bilayers and trilayers. ${ }^{132-134} \mathrm{Qu}$ and coworkers $^{135}$ reported the spontaneous formation of a partially reduced graphene oxide and PPy (prGO-PPy) film via a self oxidation-reduction strategy as shown in Fig. 13. This reaction occurs by direct exposure of the GO film to pyrrole vapor at room temperature without any extra additives. This convenient method allows for the facile preparation of asymmetric GO/ prGO-PPy hybrid films which can be applied in advanced actuation systems such as moisture or electrochemical actuators. PPy-based actuators and microactuators have many possible applications, particularly in cell biology and biomedicine because they can operate under a variety of conditions including various salt solutions, blood plasma, urine, and cell culture medium. ${ }^{136-138}$

Serpe and coworkers ${ }^{139,140}$ have demonstrated novel humidity responsive self-bending bilayer-based actuators made by depositing layers composed of poly( $N$-isopropylacrylamide)based microgels and the polyelectrolyte polydiallyldimethylammonium chloride (pDADMAC) on a flexible substrate. The responsive materials bend upon drying and the degree of bending depends on the atmospheric humidity. The dried PDADMAC layer is composed of both amorphous and crystalline phases. The amorphous layer can readily absorb water, which results in actuation, while the crystalline phase templates the specific bending characteristics of the device. They worked on applying them as artificial muscles and humidity sensors based on their understanding of the bending mechanism, which is shown in Fig. 14. To investigate the humidity response, the author connected the assembly to a circuit composed of a LED and battery and collected the LED light intensity as a function of humidity; this is shown schematically in Fig. 14b, left. They also connected the assembly to a multimeter (attached onto the gold surface of the strain sensor) and placed the setup in a humidity-controlled chamber. As can be seen in Fig. 14b, right, the light intensity likewise depended on humidity. That is, as the humidity decreased the LED light (a)

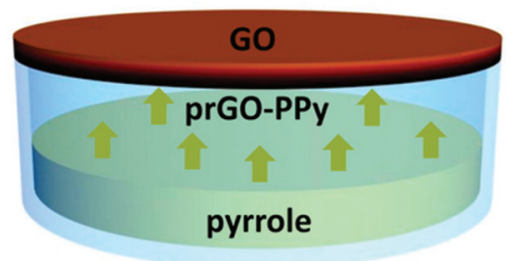

(c)

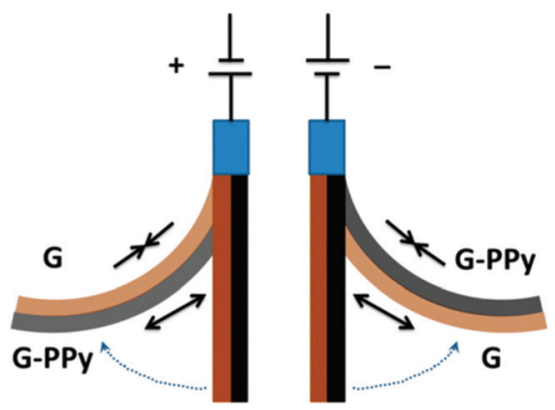

(b)

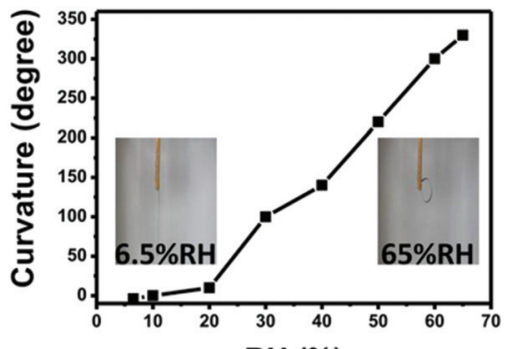

(d)

RH (\%)

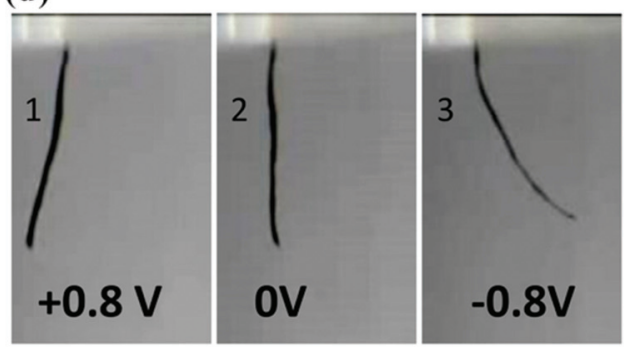

Fig. 13 (a) Schematic illustrating the fabrication process of the humidity responsive asymmetric GO/prGO-PPy film actuator. (b) Plot of the curvature of the GO/prGO-PPy film versus relative humidity $(\mathrm{RH})$. The insets are photographs of the GO/prGO-PPy film at the indicated humidities. (c) The actuation mechanism of the asymmetric G/G-PPy film. (d) Photographs of the bending for the G/G-PPy film actuator driven by the electrochemical potential within $\pm 0.8 \mathrm{~V}$. The $\mathrm{G}$ and G-PPy sides are placed on the left and right sides on the actuator, and the corresponding potentials of $+0.8 \mathrm{~V}(1), 0 \mathrm{~V}(2)$, and $-0.8 \mathrm{~V}$ (3). Reprinted with permission from ref. 135. Copyright 2016, American Chemical Society. 
a.
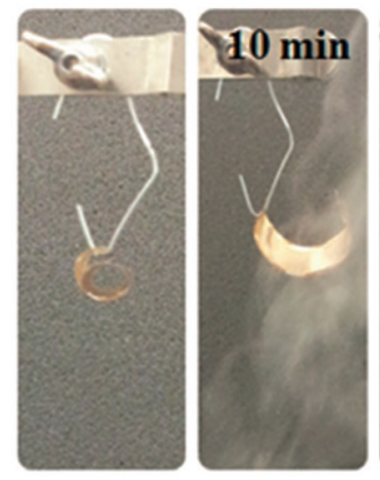

b.

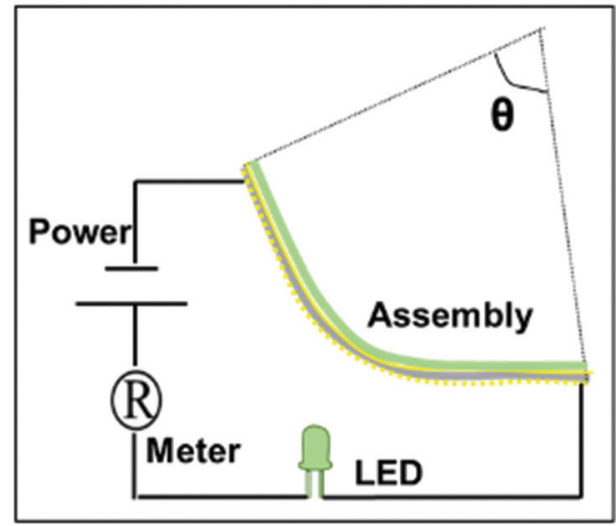

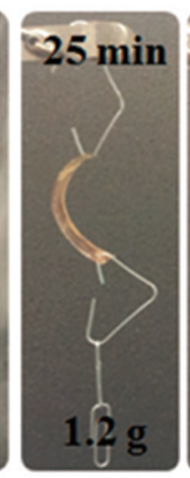
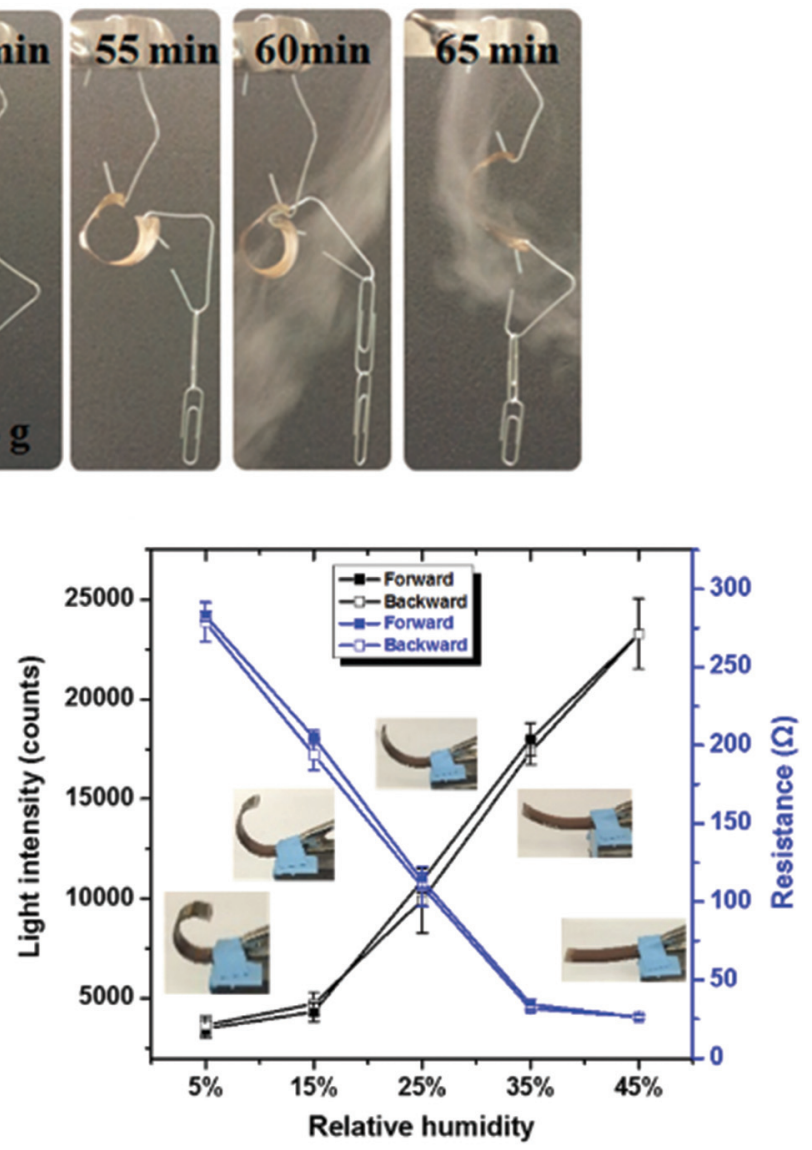

Fig. 14 (a) A bent substrate ( 2 inches long) was hung from an arm and cycled between low and high humidity. (b, left) Schematic depiction of the experimental setup used to measure resistance and LED light intensity as a function of device bending. (b, right) Light intensity (left axis) and resistance (right axis) changes induced by the bending of the bilayers coupled to the strain sensors. Reprinted with permission from ref. 139. Copyright 2013, Wiley-VCH Verlag GmbH \& Co. KGaA, Weinheim and ref. 140. Copyright 2016, Wiley-VCH Verlag GmbH \& Co. KGaA, Weinheim.

intensity increased due to the decreased resistance. There was nearly no hysteresis between multiple humidity increase and decrease cycles.

Photoresponsive polymer-based materials can also be used to generate shape memory polymers, polymer gels, and liquid crystalline polymers (LCP). ${ }^{141,142}$ They are responsible for converting light energy into mechanical work. Generally, polymers are modified with light responsive groups, which induce photochemical reactions and visible macroscopic effects on the polymer. In a specific example, photoresponsive units were used to crosslink liquid crystalline polymers and used as actuators. ${ }^{143-147}$ Warner and coworkers introduced azobenzene moieties into a monodomain nematic LCP as crosslinkers and generated films, which were shown to exhibit significant contraction upon exposure to $365 \mathrm{~nm}$ radiation. ${ }^{148}$ Terentjev and coworkers also worked on incorporating various azobenzene derivatives into LCPs and examined the deformation of the system upon exposure to UV light. ${ }^{149}$ This was demonstrated in a study published by Ikeda and coworkers. They showed that light-driven bending of azobenzene LCPs could be achieved by creating an asymmetric deformation between the surface and the bulk of a film. ${ }^{150-152}$ They found that the deformation of the polymer is dependent on the manner of alignment of the LCP mesogens. ${ }^{141}$ The monodomain LCP with in-plane alignment of mesogens bent along the alignment direction towards the irradiation source. They further fabricated light driven soft actuators from azobenzene LCP/ polyethylene laminated films. ${ }^{153}$ Recently, Bléger and coworkers described a liquid crystalline polymer film doped with visible light responsive fluorinated azobenzene capable of continuous "chaotic" oscillatory motion when exposed to ambient sunlight in air, ${ }^{154}$ which is shown in Fig. 15 . The presence of simultaneous illumination by blue and green light is necessary for the oscillating behavior to occur, suggesting that the dynamics of continuous forward and backward switching are causing the observed effect. The motion is programmed by the molecular organization in the polymer film and is independent of the position of the light source. The unique behavior makes this material potentially useful for outdoor applications including self-cleaning coatings and surfaces. Furthermore, self-propelling/morphing soft actuators could be used to harvest and convert solar energy.

Another very important class of actuators is composed of hydrogels that change volume in response to stimuli. Stimuli 
a

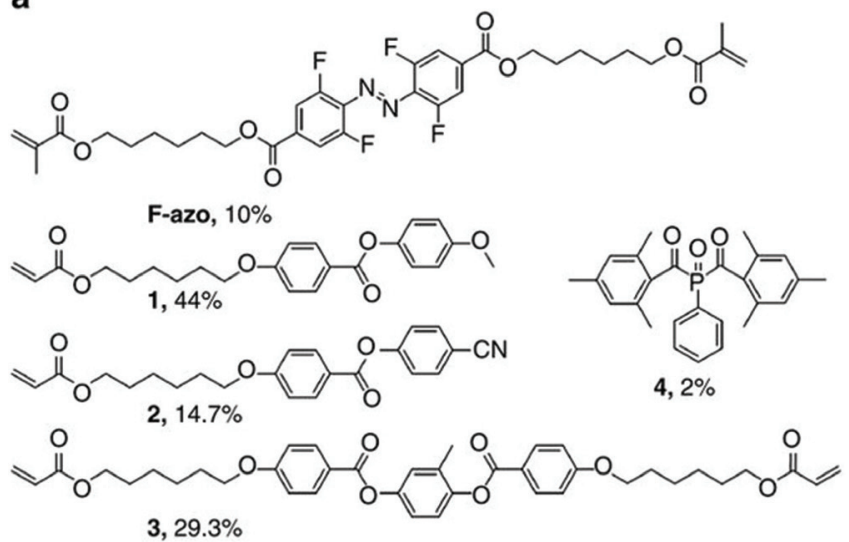

b

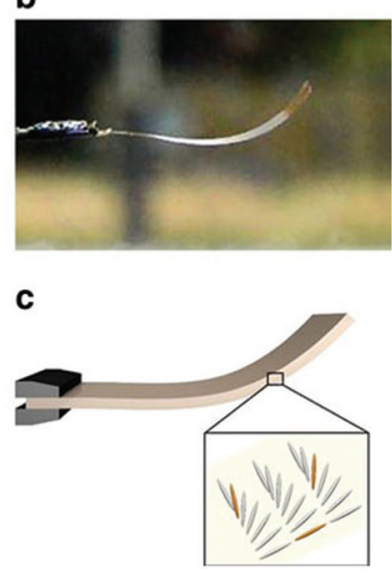

Fig. 15 (a) Chemical structures of the components and their molar percentage used to prepare the nematic liquid crystalline network. (b) Photograph of the splay-oriented film after removal from the cell under ambient interior light (homeotropic side on top) and (c) schematic of the LC splay aligned film (grey) containing F-azo molecules (orange). Reprinted with permission from ref. 154. Copyright 2016, Nature Publication.

responsive hydrogels are $3 \mathrm{D}$ networks that can absorb water and swell and shrink in response to various external stimuli including temperature, $\mathrm{pH}$, ionic strength, chemicals, electricity, and light. ${ }^{155,156}$ Hydrogel actuators produce macroscopic changes upon swelling and shrinking. ${ }^{157-160}$ In a recent example, Aida and coworkers developed a layered hydrogel consisting of cofacially oriented electrolyte nanosheets. This unusual geometry leads to significant anisotropic electrostatic repulsion in the hydrogel interior. They showed that the material could be operated by modulating its electrostatic anisotropy in response to changes of electrostatic permittivity. The electrostatic permittivity could be controlled by varying the material's solvation state, which depended on solution temperature. ${ }^{161}$ Recently, Ding and co-workers developed a computational model to demonstrate a new reversible shapechanging component design concept enabled by 3D printing of stimuli responsive polymers, shape memory polymers (SMP) and hydrogels. ${ }^{162}$ This approach uses the swelling of a hydrogel as the driving force for the shape change, and the temperature-dependent modulus of a shape memory polymer to regulate the time of such shape changes. Controlling the temperature and aqueous environment allows switching between two stable configurations. The structures are relatively stiff and can carry cargo in each state without mechanical loading for device training. By applying the design principles, they created and demonstrated the behavior of several shape-changing structures that exhibit reversible shape changes based on folding, curling, and origami concepts. Fig. 16 shows one of the examples, self-folding and unfolding flowers. The three layers were designed with different thickness ratios of layers of the elastomer, the hydrogel, and the SMP as shown in Fig. 16a. The inner layer was designed with the thinnest SMP layer of $0.3 \mathrm{~mm}$, and the second layer with a $0.4 \mathrm{~mm}$ SMP layer, and $0.5 \mathrm{~mm}$ of SMP layer for the outer layer, but the total thickness of the elastomer, the hydrogel and the SMP layer were always kept the same. The corresponding curvature change profile is
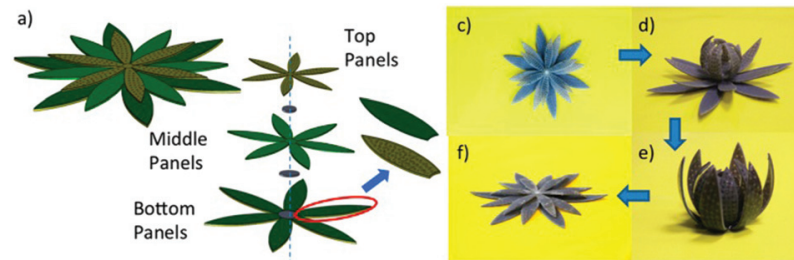

b)

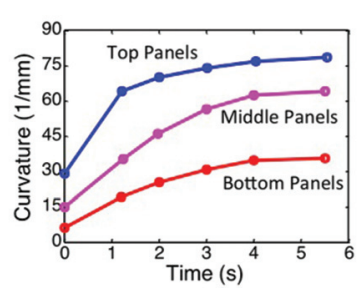

g)

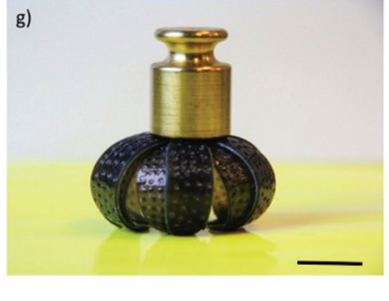

Fig. 16 A self-folding-unfolding flower. (a) Schematic of two activated shape memory petal-like structure. (b) The curvature change under thermal activation as a function of time. (c- $f$ ) The sequence of reversible actuation. (g) The dried configuration is stiff and can carry a load of $25 \mathrm{~g}$. The scale bar in ( $\mathrm{g}$ ) is $12.5 \mathrm{~mm}$. Reprinted with permission from ref. 162. Copyright 2016, Nature Publication.

shown in Fig. 16b, which has the largest curvature and the fastest response speed with the thinner SMP layer. After putting this structure into low temperature water for $12 \mathrm{~h}$, the inner layer bends a little bit as shown in Fig. 16d, and then on immersion into hot water, all layers bend and form the flowerlike structure as shown in Fig. 16e. On taking the structure out of the hot water and letting it dry, as shown in Fig. 16g, we can see that it maintains the flower shape structure and can carry a load of $25 \mathrm{~g}$. When the structure was put into hot water it became flat again as shown in Fig. 16f. The whole process can be repeated many times.

In summary, polymer-based actuators are materials capable of converting energy from external stimuli (e.g. heat, light, and electricity) to mechanical forces, thus exhibiting shape 
changes. Polymer-based actuators can be generated from a variety of materials (hydrogels, liquid crystal polymers, and shape memory polymers). The material that is used depends on the intended application, e.g., soft hydrogels could be used for delicate biological applications, while hard shape memory polymers could be used for lifting/moving heavy masses. It is this versatility of materials properties, combined with the diversity of material responsivities, that makes this research area vibrant for exploration and research.

\section{Conclusions and future perspective}

Although stimuli-responsive polymer-based systems have been known for decades, it wasn't until relatively recently that their behavior could be understood at a level that allowed their full exploitation. Stimuli-responsive polymers have been synthesized using a variety of techniques, and employed for myriad applications; we describe only some of their applications here. In the future, combining stimuli responsive polymers with biological systems, and nanoscale materials, a variety of new functions (and properties) will be accessible. This development needs to be supported by new theories that can describe the newly found behavior such that the development of new materials can be done in a smart fashion to meet the needs of a specific application. Another challenge is to develop systems that respond to multiple external stimuli in an "intelligent" and predictable manner. These materials are required to support the development of biomimetic systems with long-term stability and durability. The concepts presented in this review encompass both the polymer history introduction, and the applications of stimuli responsive materials. A more complete picture of this broad and complex topic can be obtained from the referenced articles.

\section{Acknowledgements}

MJS acknowledges funding from the University of Alberta (the Department of Chemistry and the Faculty of Science), the Natural Sciences and Engineering Research Council of Canada (NSERC), the Canada Foundation for Innovation (CFI), the Alberta Advanced Education \& Technology Small Equipment Grants Program (AET/SEGP), Grand Challenges Canada and IC-IMPACTS. YG and XL acknowledge Alberta Innovates Technology Futures for Graduate Student Scholarships.

\section{References}

1 P. J. Morris, Polymer pioneers: A popular history of the science and technology of large molecules, Chemical Heritage Foundation, Philadelphia, 2005.

2 M. R. Dove, Environ. Conserv., 1993, 20, 17-24.
3 H. Staudinger, From organic chemistry to macromolecules, Wiley, New York, 1970.

4 R. Mülhaupt, Angew. Chem., Int. Ed., 2004, 43, 1054-1063.

5 W. H. Carothers, US Pat, 2071251A, 1937.

6 W. H. Carothers, US Pat, 2130523A, 1938.

7 P. Theato, J. Polym. Sci., Part A: Polym. Chem., 2008, 46, 6677-6687.

8 A. S. Hoffman, Macromol. Symp., 1995, 98, 645-664.

9 I. Galaev and B. Mattiasson, Smart polymers: applications in biotechnology and biomedicine, CRC Press, Taylor \& Francis Group, Boca Raton, USA, 2007.

10 S. Dai, P. Ravi and K. C. Tam, Soft Matter, 2008, 4, 435449.

11 M. Heskins and J. E. Guillet, J. Macromol. Sci., Chem., 1968, 2, 1441-1455.

12 D. A. Davis, A. Hamilton, J. Yang, L. D. Cremar, D. Van Gough, S. L. Potisek, M. T. Ong, P. V. Braun, T. J. Martínez and S. R. White, Nature, 2009, 459, 68-72.

13 Y. L. Colson and M. W. Grinstaff, Adv. Mater., 2012, 24, 3878-3886.

14 T. Tanaka, I. Nishio, S.-T. Sun and S. Ueno-Nishio, Science, 1982, 218, 467-469.

15 J. Thévenot, H. Oliveira, O. Sandre and S. Lecommandoux, Chem. Soc. Rev., 2013, 42, 7099-7116.

16 M. Irie, Pure Appl. Chem., 1990, 62, 1495-1502.

17 L. Zhai, Chem. Soc. Rev., 2013, 42, 7148-7160.

18 A. Khan and J. Pessin, Diabetologia, 2002, 45, 14751483.

19 Q. M. Zhang and M. J. Serpe, in Polymer Mechanochemistry, Springer, 2015, pp. 377-424.

20 J. Hu and S. Liu, Macromolecules, 2010, 43, 8315-8330.

21 A. Bajpai, S. K. Shukla, S. Bhanu and S. Kankane, Prog. Polym. Sci., 2008, 33, 1088-1118.

22 D. Parasuraman and M. J. Serpe, ACS Appl. Mater. Interfaces, 2011, 3, 2732-2737.

23 M. Ma, L. Guo, D. G. Anderson and R. Langer, Science, 2013, 339, 186-189.

24 Q. Zhao, J. W. Dunlop, X. Qiu, F. Huang, Z. Zhang, J. Heyda, J. Dzubiella, M. Antonietti and J. Yuan, Nat. Commun., 2014, 5, 4293.

25 Z. Hu, X. Zhang and Y. Li, Science, 1995, 269, 525.

26 H. Koerner, G. Price, N. A. Pearce, M. Alexander and R. A. Vaia, Nat. Mater., 2004, 3, 115-120.

27 F. Wang, Y.-H. Lai and M.-Y. Han, Macromolecules, 2004, 37, 3222-3230.

28 F. Seker, P. R. Malenfant, M. Larsen, A. Alizadeh, K. Conway, A. M. Kulkarni, G. Goddard and R. Garaas, Adv. Mater., 2005, 17, 1941-1945.

29 G. Charlet and G. Delmas, Polymer, 1981, 22, 1181-1189.

30 H. Feil, Y. H. Bae, J. Feijen and S. W. Kim, Macromolecules, 1993, 26, 2496-2500.

31 Y. Okada and F. Tanaka, Macromolecules, 2005, 38, 44654471.

32 H. G. Schild, Prog. Polym. Sci., 1992, 17, 163-249.

33 P. Kujawa and F. M. Winnik, Macromolecules, 2001, 34, 4130-4135. 
34 D. Crespy and R. M. Rossi, Polym. Int., 2007, 56, 14611468.

35 R. Liu, M. Fraylich and B. R. Saunders, Colloid Polym. Sci., 2009, 287, 627-643.

36 Z. Song, K. Wang, C. Gao, S. Wang and W. Zhang, Macromolecules, 2016, 49, 162-171.

37 E. Karjalainen, V. Aseyev and H. Tenhu, Macromolecules, 2014, 47, 2103-2111.

38 F. A. Plamper, A. Schmalz, M. Ballauff and A. H. Müller, J. Am. Chem. Soc., 2007, 129, 14538-14539.

39 T. Thavanesan, C. Herbert and F. A. Plamper, Langmuir, 2014, 30, 5609-5619.

40 V. Bütün, S. Armes and N. Billingham, Polymer, 2001, 42, 5993-6008.

41 P. J. Roth, F. D. Jochum, F. R. Forst, R. Zentel and P. Theato, Macromolecules, 2010, 43, 4638-4645.

42 J.-F. Lutz, Ö. Akdemir and A. Hoth, J. Am. Chem. Soc., 2006, 128, 13046-13047.

43 Y. Cao, X. Zhu, J. Luo and H. Liu, Macromolecules, 2007, 40, 6481-6488.

44 I. Idziak, D. Avoce, D. Lessard, D. Gravel and X. Zhu, Macromolecules, 1999, 32, 1260-1263.

45 L. A. Connal, Q. Li, J. F. Quinn, E. Tjipto, F. Caruso and G. G. Qiao, Macromolecules, 2008, 41, 2620-2626.

46 C. D. Sorrell, M. C. Carter and M. J. Serpe, Adv. Funct. Mater., 2011, 21, 425-433.

47 M. J. Serpe, J. Kim and L. A. Lyon, Adv. Mater., 2004, 16, 184-187.

48 F. Liu and M. W. Urban, Macromolecules, 2008, 41, 65316539.

49 F.-J. Xu, E.-T. Kang and K.-G. Neoh, Biomaterials, 2006, 27, 2787-2797.

50 B.-W. Liu, H. Zhou, S.-T. Zhou, H.-J. Zhang, A.-C. Feng, C.-M. Jian, J. Hu, W.-P. Gao and J.-Y. Yuan, Macromolecules, 2014, 47, 2938-2946.

51 F. D. Jochum and P. Theato, Chem. Soc. Rev., 2013, 42, 7468-7483.

52 Y.-L. Zhao and J. F. Stoddart, Langmuir, 2009, 25, 84428446.

53 J.-F. Gohy and Y. Zhao, Chem. Soc. Rev., 2013, 42, 71177129.

54 P. D. Thornton, R. J. Mart and R. V. Ulijn, Adv. Mater., 2007, 19, 1252-1256.

55 J. Hu, G. Zhang and S. Liu, Chem. Soc. Rev., 2012, 41, 5933-5949.

56 R. V. Ulijn, J. Mater. Chem., 2006, 16, 2217-2225.

57 K. Kataoka, H. Miyazaki, M. Bunya, T. Okano and Y. Sakurai, J. Am. Chem. Soc., 1998, 120, 12694-12695.

58 Z. Gu, T. T. Dang, M. Ma, B. C. Tang, H. Cheng, S. Jiang, Y. Dong, Y. Zhang and D. G. Anderson, ACS Nano, 2013, 7, 6758-6766.

59 T. Miyata, N. Asami and T. Uragami, Nature, 1999, 399, 766-769.

60 F. Liu and M. W. Urban, Prog. Polym. Sci., 2010, 35, 3-23.

61 D. Roy, J. N. Cambre and B. S. Sumerlin, Prog. Polym. Sci., 2010, 35, 278-301.
62 M. Motornov, Y. Roiter, I. Tokarev and S. Minko, Prog. Polym. Sci., 2010, 35, 174-211.

63 I. Cobo, M. Li, B. S. Sumerlin and S. Perrier, Nat. Mater., 2015, 14, 143-159.

64 M. J. Webber, E. A. Appel, E. Meijer and R. Langer, Nat. Mater., 2016, 15, 13-26.

65 M. R. Islam, Y. Gao, X. Li, Q. M. Zhang, M. Wei and M. J. Serpe, Chin. Sci. Bull., 2014, 59, 4237-4255.

66 M. A. C. Stuart, W. T. Huck, J. Genzer, M. Müller, C. Ober, M. Stamm, G. B. Sukhorukov, I. Szleifer, V. V. Tsukruk and M. Urban, Nat. Mater., 2010, 9, 101-113.

67 M. Toma, U. Jonas, A. Mateescu, W. Knoll and J. Dostalek, J. Phys. Chem. C, 2013, 117, 11705-11712.

68 S. Maji, B. Cesur, Z. Zhang, B. G. De Geest and R. Hoogenboom, Polym. Chem., 2016, 7, 1705-1710.

69 Y. Ma, K. Promthaveepong and N. Li, Anal. Chem., 2016, 88, 8289-8293.

70 K. Paek, H. Yang, J. Lee, J. Park and B. J. Kim, ACS Nano, 2014, 8, 2848-2856.

71 A. Seeboth, D. Lötzsch, R. Ruhmann and O. Muehling, Chem. Rev., 2014, 114, 3037-3068.

72 J. H. Holtz and S. A. Asher, Nature, 1997, 389, 829-832.

73 S. A. Asher, K. W. Kimble and J. P. Walker, Chem. Mater., 2008, 20, 7501-7509.

74 J. H. Holtz, J. S. Holtz, C. H. Munro and S. A. Asher, Anal. Chem., 1998, 70, 780-791.

75 K. Lee and S. A. Asher, J. Am. Chem. Soc., 2000, 122, 95349537.

76 C. E. Reese, M. E. Baltusavich, J. P. Keim and S. A. Asher, Anal. Chem., 2001, 73, 5038-5042.

77 J. P. Walker and S. A. Asher, Anal. Chem., 2005, 77, 15961600.

78 J. M. Weissman, H. B. Sunkara, A. S. Tse and S. A. Asher, Thermally Switchable Periodicities and Diffraction from Novel Mesoscopically Ordered Materials, DTIC Document, 1996.

79 M. C. Chiappelli and R. C. Hayward, Adv. Mater., 2012, 24, 6100-6104.

80 K. C. Johnson, F. Mendez and M. J. Serpe, Anal. Chim. Acta, 2012, 739, 83-88.

81 Q. M. Zhang, X. Li, M. R. Islam, M. Wei and M. J. Serpe, J. Mater. Chem. C, 2014, 2, 6961-6965.

82 W. Xu, Y. Gao and M. J. Serpe, J. Mater. Chem. C, 2014, 2, 3873-3878.

83 Q. M. Zhang, W. Xu and M. J. Serpe, Angew. Chem., Int. Ed., 2014, 53, 4827-4831.

84 M. R. Islam and M. J. Serpe, Biosens. Bioelectron., 2013, 49, 133-138.

85 C. D. Sorrell and M. J. Serpe, Anal. Bioanal. Chem., 2012, 402, 2385-2393.

86 Q. M. Zhang, A. Ahiabu, Y. Gao and M. J. Serpe, J. Mater. Chem. C, 2015, 3, 495-498.

87 Q. M. Zhang, D. Berg, S. M. Mugo and M. J. Serpe, Chem. Commun., 2015, 51, 9726-9728.

88 M. R. Islam and M. J. Serpe, Chem. Commun., 2013, 49, 2646-2648. 
89 M. R. Islam and M. J. Serpe, Anal. Bioanal. Chem., 2014, 406, 4777-4783.

90 Q. M. Zhang, W. Wang, Y.-Q. Su, E. J. Hensen and M. J. Serpe, Chem. Mater., 2016, 28, 259-265.

91 M. B. Yatvin, J. N. Weinstein, W. H. Dennis and R. Blumenthal, Science, 1978, 202, 1290-1293.

92 A. P. Blum, J. K. Kammeyer, A. M. Rush, C. E. Callmann, M. E. Hahn and N. C. Gianneschi, J. Am. Chem. Soc., 2015, 137, 2140-2154.

93 J. K. Oh, D. I. Lee and J. M. Park, Prog. Polym. Sci., 2009, 34, 1261-1282.

94 S. Mura, J. Nicolas and P. Couvreur, Nat. Mater., 2013, 12, 991-1003.

95 M. Bernadete Riemma Pierre and F. Cristina Rossetti, Curr. Drug Targets, 2014, 15, 281-291.

96 X. Hong, Z. Wu, L. Chen, F. Wu, L. Wei and W. Yuan, Nano-Micro Lett., 2014, 6, 191-199.

97 R. F. Donnelly, T. R. R. Singh, M. J. Garland, K. Migalska, R. Majithiya, C. M. McCrudden, P. L. Kole, T. M. T. Mahmood, H. O. McCarthy and A. D. Woolfson, Adv. Funct. Mater., 2012, 22, 4879-4890.

98 Y. Deng, J. Chen, Y. Zhao, X. Yan, L. Zhang, K. Choy, J. Hu, H. J. Sant, B. K. Gale and T. Tang, Sci. Rep., 2016, 6, 30430.

99 R. F. Donnelly, M. T. McCrudden, A. Z. Alkilani, E. Larrañeta, E. McAlister, A. J. Courtenay, M.-C. Kearney, T. R. R. Singh, H. O. McCarthy and V. L. Kett, PLoS One, 2014, 9, e111547.

100 M. J. Garland, E. Caffarel-Salvador, K. Migalska, A. D. Woolfson and R. F. Donnelly, J. Controlled Release, 2012, 159, 52-59.

101 E. M. Cahill and E. D. O'Cearbhaill, Bioconjugate Chem., 2015, 26, 1289-1296.

102 J. G. Hardy, E. Larraneta, R. F. Donnelly, N. McGoldrick, K. Migalska, M. T. McCrudden, N. J. Irwin, L. Donnelly and C. P. McCoy, Mol. Pharm., 2016, 13, 907-914.

103 S. Y. Yang, E. D. O’Cearbhaill, G. C. Sisk, K. M. Park, W. K. Cho, M. Villiger, B. E. Bouma, B. Pomahac and J. M. Karp, Nat. Commun., 2013, 4, 1702.

104 M.-C. Chen, Z.-W. Lin and M.-H. Ling, ACS Nano, 2015, 10, 93-101.

105 M.-C. Chen, M.-H. Ling, K.-W. Wang, Z.-W. Lin, B.-H. Lai and D.-H. Chen, Biomacromolecules, 2015, 16, 1598-1607.

106 Y. Ye, J. Yu, C. Wang, N. Y. Nguyen, G. M. Walker, J. B. Buse and Z. Gu, Adv. Mater., 2016, 28, 3115-3121.

107 J. Yu, Y. Zhang, Y. Ye, R. DiSanto, W. Sun, D. Ranson, F. S. Ligler, J. B. Buse and Z. Gu, Proc. Natl. Acad. Sci. U. S. A., 2015, 112, 8260-8265.

108 C. Wang, Y. Ye, G. M. Hochu, H. Sadeghifar and Z. Gu, Nano Lett., 2016, 16, 2334-2340.

109 J. K. Oh, R. Drumright, D. J. Siegwart and K. Matyjaszewski, Prog. Polym. Sci., 2008, 33, 448-477.

110 H. Bysell, R. Månsson, P. Hansson and M. Malmsten, Adv. Drug Delivery Rev., 2011, 63, 1172-1185.

111 N. Smeets and T. Hoare, J. Polym. Sci., Part A: Polym. Chem., 2013, 51, 3027-3043.
112 Y. Gao, G. P. Zago, Z. Jia and M. J. Serpe, ACS Appl. Mater. Interfaces, 2013, 5, 9803-9808.

113 S. Guo, Y. Gao, M. Wei, Q. M. Zhang and M. J. Serpe, J. Mater. Chem. B, 2015, 3, 2516-2521.

114 Y. Gao, K. Y. Wong, A. Ahiabu and M. J. Serpe, J. Mater. Chem. B, 2016, 4, 5144-5150.

115 Y. Gao, A. Ahiabu and M. J. Serpe, ACS Appl. Mater. Interfaces, 2014, 6, 13749-13756.

116 Y. Gao, W. Xu and M. J. Serpe, J. Mater. Chem. C, 2014, 2, 5878-5884.

117 M. Topp, P. Dijkstra, H. Talsma and J. Feijen, Macromolecules, 1997, 30, 8518-8520.

118 J. Virtanen, S. Holappa, H. Lemmetyinen and H. Tenhu, Macromolecules, 2002, 35, 4763-4769.

119 R. Motokawa, K. Morishita, S. Koizumi, T. Nakahira and M. Annaka, Macromolecules, 2005, 38, 5748-5760.

120 W. Zhang, L. Shi, K. Wu and Y. An, Macromolecules, 2005, 38, 5743-5747.

121 S. Qin, Y. Geng, D. E. Discher and S. Yang, Adv. Mater., 2006, 18, 2905-2909.

122 X. Wang, J. Hu, G. Liu, J. Tian, H. Wang, M. Gong and S. Liu, J. Am. Chem. Soc., 2015, 137, 15262-15275.

123 X. Chi, X. Ji, D. Xia and F. Huang, J. Am. Chem. Soc., 2015, 137, 1440-1443.

124 Y. Wang, Q. Luo, W. Zhu, X. Li and Z. Shen, Polym. Chem., 2016, 7, 2665-2673.

125 S. Y. An, S. H. Hong, C. Tang and J. K. Oh, Polym. Chem., 2016, 7, 4751-4760.

126 T. Otero and J. Sansinena, Bioelectrochem. Bioenerg., 1997, 42, 117-122.

127 W. Takashima, M. Kaneko, K. Kaneto and A. G. MacDiarmid, Synth. Met., 1995, 71, 2265-2266.

128 Q. Pei and O. Inganläs, Adv. Mater., 1992, 4, 277-278.

129 Q. Pei and O. Inganäs, Synth. Met., 1993, 57, 37183723.

130 K. Kaneto, M. Kaneko, Y. Min and A. G. MacDiarmid, Synth. Met., 1995, 71, 2211-2212.

131 A. MacDiarmid, K. Kaneto, H. Saito and Y. Min, Polym. Mater. Sci. Eng., 1994, 71, 713-714.

132 T. F. N. Otero and M. T. Cortes, Adv. Mater., 2003, 15, 279282.

133 F. García-Córdova, L. Valero, Y. A. Ismail and T. F. Otero, J. Mater. Chem., 2011, 21, 17265-17272.

134 T. F. Otero, J. J. Sanchez and J. G. Martinez, J. Phys. Chem. $B, 2012,116,5279-5290$.

135 Y. Jiang, C. Hu, H. Cheng, C. Li, T. Xu, Y. Zhao, H. Shao and L. Qu, ACS Nano, 2016, 10, 4735-4741.

136 K. Svennersten, M. Berggren, A. Richter-Dahlfors and E. W. Jager, Lab Chip, 2011, 11, 3287-3293.

137 E. W. Jager, C. Immerstrand, K.-E. Magnusson, O. Inganas and I. Lundstrom, Biomedical applications of polypyrrole microactuators: from single-cell clinic to microrobots, in 1st Annual International Conference on Microtechnologies in Medicine and Biology, 2000.

138 E. W. Jager, O. Inganäs and I. Lundström, Science, 2000, 288, 2335-2338. 
139 M. R. Islam, X. Li, K. Smyth and M. J. Serpe, Angew. Chem., Int. Ed., 2013, 52, 10330-10333.

140 X. Li and M. J. Serpe, Adv. Funct. Mater., 2016, 26, 32823290.

141 J. Wei and Y. Yu, Soft Matter, 2012, 8, 8050-8059.

142 H. Jiang, S. Kelch and A. Lendlein, Adv. Mater., 2006, 18, 1471-1475.

143 J. Garcia-Amorós, A. Piñol, H. Finkelmann and D. Velasco, Org. Lett., 2011, 13, 2282-2285.

144 T. J. White, N. V. Tabiryan, S. V. Serak, U. A. Hrozhyk, V. P. Tondiglia, H. Koerner, R. A. Vaia and T. J. Bunning, Soft Matter, 2008, 4, 1796-1798.

145 T. J. White, S. V. Serak, N. V. Tabiryan, R. A. Vaia and T. J. Bunning, J. Mater. Chem., 2009, 19, 1080-1085.

146 K. M. Lee, M. L. Smith, H. Koerner, N. Tabiryan, R. A. Vaia, T. J. Bunning and T. J. White, Adv. Funct. Mater., 2011, 21, 2913-2918.

147 M. Camacho-Lopez, H. Finkelmann, P. Palffy-Muhoray and M. Shelley, Nat. Mater., 2004, 3, 307-310.

148 H. Finkelmann, E. Nishikawa, G. Pereira and M. Warner, Phys. Rev. Lett., 2001, 87, 015501.

149 P. Hogan, A. Tajbakhsh and E. Terentjev, Phys. Rev. E: Stat. Phys., Plasmas, Fluids, Relat. Interdiscip. Top., 2002, 65, 041720 .

150 T. Ikeda, M. Nakano, Y. Yu, O. Tsutsumi and A. Kanazawa, Adv. Mater., 2003, 15, 201-205.
151 Y. Yu, M. Nakano and T. Ikeda, Nature, 2003, 425, 145145.

152 M. Kondo, Y. Yu and T. Ikeda, Angew. Chem., Int. Ed., 2006, 118, 1406-1410.

153 M. Yamada, M. Kondo, J. I. Mamiya, Y. Yu, M. Kinoshita, C. J. Barrett and T. Ikeda, Angew. Chem., Int. Ed., 2008, 47, 4986-4988.

154 K. Kumar, C. Knie, D. Bléger, M. A. Peletier, H. Friedrich, S. Hecht, D. J. Broer, M. G. Debije and A. P. Schenning, Nat. Commun., 2016, 7, 11975.

155 M. C. Koetting, J. T. Peters, S. D. Steichen and N. A. Peppas, Mater. Sci. Eng., R, 2015, 93, 1-49.

156 Y. Qiu and K. Park, Adv. Drug Delivery Rev., 2012, 64, 49-60.

157 N. Bassik, B. T. Abebe, K. E. Laflin and D. H. Gracias, Polymer, 2010, 51, 6093-6098.

158 R. Luo, J. Wu, N. D. Dinh and C. H. Chen, Adv. Funct. Mater., 2015, 25, 7272-7279.

159 G. H. Kwon, Y. Y. Choi, J. Y. Park, D. H. Woo, K. B. Lee, J. H. Kim and S.-H. Lee, Lab Chip, 2010, 10, 1604-1610.

160 I. Tokarev and S. Minko, Soft Matter, 2009, 5, 511-524.

161 Y. S. Kim, M. Liu, Y. Ishida, Y. Ebina, M. Osada, T. Sasaki, T. Hikima, M. Takata and T. Aida, Nat. Mater., 2015, 14, 1002-1007.

162 Y. Mao, Z. Ding, C. Yuan, S. Ai, M. Isakov, J. Wu, T. Wang, M. L. Dunn and H. J. Qi, Sci. Rep., 2016, 6, 24761. 